\title{
Electroweak phase transition in non-minimal Higgs sectors
}

\author{
Philipp Basler, Margarete Mühlleitner and Jonas Müller \\ Institute for Theoretical Physics, Karlsruhe Institute of Technology, \\ 76128 Karlsruhe, Germany \\ E-mail: philipp.basler@kit.edu, margarete.muehlleitner@kit.edu, \\ jonas.mueller@kit.edu
}

AbStRaCT: Higgs sector extensions beyond the Standard Model (BSM) provide additional sources of $\mathrm{CP}$ violation and further scalar states that help to trigger a strong first order electroweak phase transition (SFOEWPT) required to generate the observed baryon asymmetry of the Universe through electroweak baryogenesis. We investigate the CP-violating 2-Higgs-Doublet Model (C2HDM) and the Next-to-Minimal 2-Higgs-Doublet Model (N2HDM) with respect to their potential to generate an SFOEWPT while being compatible with all relevant and recent theoretical and experimental constraints. The implications of an SFOEWPT on the collider phenomenology of the two models are analysed in detail in particular with respect to Higgs pair production. We provide benchmark points for parameter points that are compatible with an SFOEWPT and provide distinct di-Higgs signatures.

Keywords: Beyond Standard Model, CP violation, Higgs Physics

ArXiv EPRINT: 1912.10477 


\section{Contents}

1 Introduction 1

2 The effective potential 3

2.1 The tree-level C2HDM potential 3

2.2 The tree-level N2HDM potential 6

2.3 One-loop effective potential at finite temperature 8

3 Renormalisation $\quad 10$

4 Numerical analysis $\quad 12$

$\begin{array}{lll}4.1 & \text { Minimisation of the effective potential } & 12\end{array}$

$\begin{array}{lll}4.2 & \text { Constraints and parameter scan } & 12\end{array}$

5 Results $\quad 16$

$\begin{array}{lll}5.1 & \text { C2HDM - update } & 17\end{array}$

$\begin{array}{lll}5.2 & \text { N2HDM - phenomenology of the SFOEWPT } & 19\end{array}$

5.2.1 Mass spectrum of the N2HDM T1 20

5.2.2 N2HDM T1 - trilinear Higgs self-couplings 23

5.2.3 N2HDM T1 — benchmark points 25

5.2.4 Mass spectrum of the N2HDM T2 28

5.2.5 N2HDM T2 - trilinear Higgs self-couplings 30

5.3 Comparison of the C2HDM and the N2HDM di-Higgs rates 31

5.3.1 C2HDM and N2HDM T1 31

5.3.2 C2HDM and N2HDM T2 34

6 Conclusions $\quad 35$

\section{Introduction}

The discovery of the Higgs boson by the LHC experiments ATLAS [1] and CMS [2] has been a great success for particle physics. While the Higgs boson behaves very Standard Model (SM)-like [3-6] it is clear that there must be physics beyond the SM (BSM) in order to solve the remaining puzzles that cannot be explained within the SM. Thus the observed baryon asymmetry of the Universe [7] calls for new physics extensions. Electroweak baryogenesis [8-16] provides a mechanism for its generation if all three Sakharov conditions [17] are fulfilled. These are baryon number violation, $\mathrm{C}$ and $\mathrm{CP}$ violation and departure from the thermal equilibrium. The asymmetry can be generated if the electroweak phase transition (EWPT) is of strong first order $[14,16]$. The strong first order 
phase transition proceeds through bubble formation and suppresses the baryon number violating sphaleron transitions in the false vacuum $[18,19]$. While the SM in principle fulfills all three Sakharov conditions the EWPT is not of strong first order [20-23]. This would require an SM Higgs boson mass of around $70-80 \mathrm{GeV}[24,25]$ in contrast to the mass value of $125 \mathrm{GeV}$ of the discovered Higgs boson [26]. Moreover, the SM CP violation arising from the Cabibbo-Kobayashi-Maskawa (CKM) matrix is not large enough [16, 27-29]. Extended Higgs sectors beyond the SM provide additional sources of $\mathrm{CP}$ violation and further scalar states that help to trigger a strong first order EWPT (SFOEWPT). Thus previous studies within the 2-Higgs-Doublet Model (2HDM) [30, 31], which belongs to the simplest BSM extensions, have shown that it provides a framework where an SFOEWPT can be realized in accordance with the relevant theoretical and experimental constraints, both in the CP-conserving [32-51] and in the CP-violating case [52-61].

With the increasing precision in the Higgs property measurements new physics extensions get more and more constrained and also models being so far successful in generating the observed baryon-antibaryon asymmetry get more and more under tension. In view of this situation, we first of all revisit our results of ref. [60], where we investigated the CP-violating 2HDM (C2HDM) [62] with respect to an SFOEWPT, by taking into account the newest collider constraints. We then move on to the Next-to-Minimal 2HDM (N2HDM) $[63,64]$ in order to investigate the question if it can provide an SFOEWPT and how this connects with collider phenomenology. The N2HDM is based on the extension of the CP-conserving 2HDM by a real singlet scalar field, inducing a Higgs sector consisting of three scalar, one pseudoscalar and two charged Higgs boson fields. The C2HDM and N2HDM resemble each other in the sense that they both provide at least three neutral Higgs bosons. While in the C2HDM their phenomenology is determined by the amount of $\mathrm{CP}$ admixture to the mass eigenstates, in the N2HDM it is their singlet admixture that governs phenomenology. Moreover, the N2HDM features more input parameters that can be tuned to render the model compatible with all theoretical and experimental constraints, so that it provides more flexibility in finding parameter points that are both compatible with these constraints and provide an SFOEWPT. We compare both models, by taking into account all relevant and recent theoretical and experimental constraints, with respect to their ability to provide an SFOEWPT. We investigate the potential to distinguish between both models in view of these requirements. In particular, the size of the trilinear Higgs self-couplings that is necessary for an SFOEWPT is analysed and their implication for Higgs pair production. We provide benchmark points that connect the requirement of an SFOEWPT with specific features in the collider phenomenology, in particular Higgs pair production. Both models are interesting here also because their non-minimal Higgs sectors allow for the production of mixed Higgs pair final states. With our analysis we hope to provide a link between collider phenomenology and cosmology.

The paper is organized as follows. In section 2 we introduce the computation of the effective potential in the C2HDM and N2HDM and set our notation. In section 3, we describe the renormalisation of the N2HDM that is new. The one for the C2HDM has been provided previously in [60]. The basics of our numerical analysis are introduced in section 4 . Section 5 is devoted to the presentation of our results, where we first give in subsection 5.1 
an update of the C2HDM by including the newest constraints. We then move on to the detailed presentation of the N2HDM phenomenology of the SFOEWPT in subsection 5.2, both discussing the related mass spectra and trilinear Higgs self-couplings and providing benchmark points. In subsection 5.3 we compare the C2HDM and N2HDM rates for Higgs pair production induced by the requirement of an SFOEWPT. Our conclusions are given in section 6.

\section{The effective potential}

In order to decide if the EWPT is of strong first order, we have to determine the value $v_{c}$ of the VEV at the critical temperature $T_{c}$. The VEV at the temperature $T$ is given by the minimum of the one-loop corrected effective potential at non-zero temperature $T$. In this section we provide the loop-corrected effective potentials at finite temperature for our two models under investigation, the C2HDM and the N2HDM. We start with the tree-level potentials and thereby set our notation.

\subsection{The tree-level C2HDM potential}

We briefly introduce the C2HDM Higgs sector and refer to [60, 65] for a more detailed introduction. In $2 \mathrm{HDM}$ s $[30,31]$ the SM Higgs potential is extended by an additional $\mathrm{SU}(2)_{L}$ scalar doublet yielding the Higgs potential,

$$
\begin{aligned}
V_{\mathrm{C} 2 \mathrm{HDM}}= & m_{11}^{2} \Phi_{1}^{\dagger} \Phi_{1}+m_{22}^{2} \Phi_{2}^{\dagger} \Phi_{2}+\frac{\lambda_{1}}{2}\left(\Phi_{1}^{\dagger} \Phi_{1}\right)^{2}+\frac{\lambda_{2}}{2}\left(\Phi_{1}^{\dagger} \Phi_{1}\right)^{2}+\lambda_{3}\left(\Phi_{1}^{\dagger} \Phi_{1}\right)\left(\Phi_{2}^{\dagger} \Phi_{2}\right) \\
& +\lambda_{4}\left(\Phi_{1}^{\dagger} \Phi_{2}\right)^{2}+\left[\frac{\lambda_{5}}{2}\left(\Phi_{1}^{\dagger} \Phi_{2}\right)^{2}-m_{12}^{2}\left(\Phi_{1}^{\dagger} \Phi_{2}\right)+\text { h.c. }\right]
\end{aligned}
$$

with a softly broken discrete $\mathbb{Z}_{2}$ symmetry under which $\Phi_{1} \rightarrow \Phi_{1}, \Phi_{2} \rightarrow-\Phi_{2}$, which ensures the absence of tree-level flavour-changing neutral currents (FCNC) upon extension to the Yukawa sector. The hermiticity of the Lagrangian requires all couplings to be real except for $m_{12}^{2}$ and $\lambda_{5}$. If their complex phases are unrelated the model is CP-violating and called C2HDM [62]. In the following, we will adopt the conventions of refs. [65, 66]. We denote the VEVs of the EW minimum by $\omega_{i} \in \mathbb{R}(i=1,2)$ and write the two Higgs doublets as expansions around the VEVs in terms of the charged field components $\rho_{i}$ and $\eta_{i}$ and the neutral CP-even and CP-odd fields $\zeta_{i}$ and $\psi_{i}$. At tree level, the general vacuum structure of the $2 \mathrm{HDM}$ allows for three different possible vacua that are given by the normal EW-breaking vacuum, a CP-breaking and a charge-breaking $(\mathrm{CB})$ vacuum. It has been shown that vacua breaking different symmetries cannot coexist at tree level in the 2HDM [67-69]. Since this statement might not hold at higher orders, or be broken by finite temperature effects, we allow for a more general vacuum structure. We therefore include the possibility of a CB- and CP-breaking VEV in the field expansion, denoted by $\omega_{\mathrm{CB}}$ and $\omega_{\mathrm{CP}}$, respectively,

$$
\Phi_{1}=\frac{1}{\sqrt{2}}\left(\begin{array}{c}
\rho_{1}+\mathrm{i} \eta_{1} \\
\zeta_{1}+\omega_{1}+\mathrm{i} \psi_{1}
\end{array}\right) \quad \Phi_{2}=\frac{1}{\sqrt{2}}\left(\begin{array}{c}
\rho_{2}+\omega_{\mathrm{CB}}+\mathrm{i} \eta_{2} \\
\zeta_{2}+\omega_{2}+\mathrm{i}\left(\psi_{2}+\omega_{\mathrm{CP}}\right)
\end{array}\right)
$$


with

$$
\left\langle\Phi_{1}\right\rangle=\frac{1}{\sqrt{2}}\left(\begin{array}{c}
0 \\
\omega_{1}
\end{array}\right) \text { and }\left\langle\Phi_{2}\right\rangle=\frac{1}{\sqrt{2}}\left(\begin{array}{c}
\omega_{\mathrm{CB}} \\
\omega_{2}+\mathrm{i} \omega_{\mathrm{CP}}
\end{array}\right),
$$

where the brackets around the doublets stand for their vacuum state. The VEVs of our present vacuum at zero temperature are denoted by

$$
\left.v_{i} \equiv \omega_{i}\right|_{T=0} \quad i=1,2, \mathrm{CP}, \mathrm{CB},
$$

with

$$
v_{\mathrm{CP}}=v_{\mathrm{CB}} \equiv 0 .
$$

A non-zero CB VEV would break the conservation of electric charge and introduce massive photons so that we will neglect in our analysis parameter points evolving such unphysical vacuum structures. The VEVs of the normal EW minimum are related to the SM VEV $v \approx 246 \mathrm{GeV}$ by

$$
v_{1}^{2}+v_{2}^{2} \equiv v^{2} .
$$

The angle $\beta$ is defined by the ratio of $v_{1}$ and $v_{2}$,

$$
\tan \beta=\frac{v_{2}}{v_{1}} .
$$

Using the minimum condition of the potential

$$
\left.\frac{\partial V_{\text {tree }}}{\partial \Phi_{i}^{\dagger}}\right|_{\Phi_{j}=\left\langle\Phi_{j}\right\rangle} \stackrel{!}{=} 0, \quad i, j \in\{1,2\}
$$

yields

$$
\begin{aligned}
m_{11}^{2} & =\operatorname{Re} m_{12}^{2} \frac{v_{2}}{v_{1}}-\frac{\lambda_{1}}{2} v_{1}^{2}-\frac{\lambda_{3}+\lambda_{4}+\operatorname{Re} \lambda_{5}}{2} v_{2}^{2} \\
m_{22}^{2} & =\operatorname{Re} m_{12}^{2} \frac{v_{1}}{v_{2}}-\frac{\lambda_{2}}{2} v_{2}^{2}-\frac{\lambda_{3}+\lambda_{4}+\operatorname{Re} \lambda_{5}}{2} v_{1}^{2} \\
\operatorname{Im} m_{12}^{2} & =\operatorname{Im} \lambda_{5} \frac{v_{1} v_{2}}{2} .
\end{aligned}
$$

We use eq. (2.9a) and eq. (2.9b) to trade $m_{11}^{2}$ and $m_{22}^{2}$ for $v_{1}$ and $v_{2}$. The two possible sources of $\mathrm{CP}$ violation are related to each other through eq. (2.9c) so that one independent $\mathrm{CP}$-violating phase remains in the C2HDM. Rotating the fields $\psi_{1}$ and $\psi_{2}$ with $\beta$ yields

$$
\left(\begin{array}{l}
G^{0} \\
\zeta_{3}
\end{array}\right)=\left(\begin{array}{cc}
\cos \beta & \sin \beta \\
-\sin \beta & \cos \beta
\end{array}\right)\left(\begin{array}{l}
\psi_{1} \\
\psi_{2}
\end{array}\right)
$$

where $G^{0}$ denotes the neutral Goldstone boson. The three neutral mass eigenstates $H_{k}$ $(k=1,2,3)$ of the $\mathrm{C} 2 \mathrm{HDM}$ are obtained by the rotation of the gauge eigenstates $\zeta_{k}$ $(k=1,2,3)$ to the mass basis,

$$
\left(\begin{array}{l}
H_{1} \\
H_{2} \\
H_{3}
\end{array}\right)=R\left(\begin{array}{l}
\zeta_{1} \\
\zeta_{2} \\
\zeta_{3}
\end{array}\right)
$$




\begin{tabular}{|cccc|ccccc|}
\hline & $u$-type & $d$-type & leptons & $\mathrm{Q}$ & $u_{R}$ & $d_{R}$ & $\mathrm{~L}$ & $l_{R}$ \\
\hline Type I & $\Phi_{2}$ & $\Phi_{2}$ & $\Phi_{2}$ & + & - & - & + & - \\
Type II & $\Phi_{2}$ & $\Phi_{1}$ & $\Phi_{1}$ & + & - & + & + & - \\
lepton-specific & $\Phi_{2}$ & $\Phi_{2}$ & $\Phi_{1}$ & + & - & + & + & - \\
flipped & $\Phi_{2}$ & $\Phi_{1}$ & $\Phi_{2}$ & + & - & - & + & + \\
\hline
\end{tabular}

Table 1. Left: definition of the $2 \mathrm{HDM}$ types through the allowed couplings among fermions and Higgs doublets. Right: corresponding $\mathbb{Z}_{2}$ parity assignments to the left-handed quark and lepton doublets, $Q, L$, and the right-handed singlets of the up-type and down-type quarks, $u_{R}$ and $d_{R}$, and right-handed leptons $l_{R}$.

with the rotation matrix $R$ parametrized in terms of the mixing angles $\alpha_{k}$ as $\left(\cos \alpha_{k} \equiv c_{k}\right.$, $\left.\sin \alpha_{k} \equiv s_{k}\right)$

$$
R=\left(\begin{array}{ccc}
c_{1} c_{2} & s_{1} c_{2} & s_{s} \\
-\left(c_{1} s_{2} s_{3}+s_{1} c_{3}\right) & c_{1} c_{3}-s_{1} s_{2} s_{3} & c_{2} s_{3} \\
-c_{1} s_{2} c_{3}+s_{1} s_{3} & -\left(c_{1} s_{3}+s_{1} s_{2} c_{3}\right) & c_{2} c_{3}
\end{array}\right)
$$

Without loss of generality, the rotation angles $\alpha_{1,2,3}$ can be chosen in the interval

$$
-\frac{\pi}{2} \leq \alpha_{i}<\frac{\pi}{2}
$$

The mass eigenvalues are given by

$$
R M_{\text {Scalar }}^{2} R^{T}=\operatorname{diag}\left(m_{H_{1}}^{2}, m_{H_{2}}^{2}, m_{H_{3}}^{2}\right) .
$$

The mass eigenstates are ordered by ascending masses as

$$
m_{H_{1}} \leq m_{H_{2}} \leq m_{H_{3}}
$$

Using the minimum conditions and the rotation to the mass eigenstates, the following set of nine independent parameters of the C2HDM remains [70],

$$
v, \quad \tan \beta, \quad \alpha_{1,2,3}, \quad m_{H_{i}}, \quad m_{H_{j}}, \quad m_{H^{ \pm}} \quad \text { and } \operatorname{Re}\left(m_{12}^{2}\right) .
$$

The $m_{H_{i}}$ and $m_{H_{j}}$ denote any of the masses of two among the three neutral Higgs bosons. The third neutral Higgs boson mass is determined through

$$
\sum_{k=1}^{3} m_{H_{k}}^{2} R_{k 3}\left(R_{k 2} \tan \beta-R_{k 1}\right)=0 .
$$

One of the three Higgs bosons is identified with the SM-like Higgs boson with the measured mass value of $m_{h}=125.09 \mathrm{GeV}$ [26]. As mentioned above, extending the $\mathbb{Z}_{2}$ symmetry to the Yukawa sector ensures that each type of the up- and down-type quarks and charged leptons can couple to only one of the two Higgs doublets so that FCNCs are avoided at tree level. In table 1 the different types of the 2HDM, type I, type II, lepton-specific and flipped, are listed. In this analysis we will concentrate on type I and type II. 


\subsection{The tree-level N2HDM potential}

We give a brief introduction in the N2HDM and refer for more details to [64]. The treelevel potential of the N2HDM consists of a CP-conserving $2 \mathrm{HDM}$ which is extended by a real singlet field $\Phi_{S}$. The potential is invariant under two discrete $\mathbb{Z}_{2}$ symmetries. We call the one given by the generalisation of the $2 \mathrm{HDM}$ symmetry to avoid tree-level FCNCs $\mathbb{Z}_{2}$ under which

$$
\Phi_{1} \rightarrow \Phi_{1}, \quad \Phi_{2} \rightarrow-\Phi_{2}, \quad \Phi_{S} \rightarrow \Phi_{S}
$$

We call the second one $\mathbb{Z}_{2}^{\prime}$. It is defined as

$$
\Phi_{1} \rightarrow \Phi_{1}, \quad \Phi_{2} \rightarrow \Phi_{2}, \quad \Phi_{S} \rightarrow-\Phi_{S}
$$

The most general tree-level potential invariant under these transformations apart from a term proportional to $m_{12}^{2}$ that softly breaks $\mathbb{Z}_{2}$, reads [64]

$$
\begin{aligned}
V_{\mathrm{N} 2 \mathrm{HDM}}= & m_{11}^{2} \Phi_{1}^{\dagger} \Phi_{1}+m_{22}^{2} \Phi_{2}^{\dagger} \Phi_{2}-m_{12}^{2}\left(\Phi_{1}^{\dagger} \Phi_{2}+\text { h.c. }\right)+\frac{\lambda_{1}}{2}\left(\Phi_{1}^{\dagger} \Phi_{1}\right)^{2}+\frac{\lambda_{2}}{2}\left(\Phi_{2}^{\dagger} \Phi_{2}\right)^{2} \\
& +\lambda_{3} \Phi_{1}^{\dagger} \Phi_{1} \Phi_{2}^{\dagger} \Phi_{2}+\lambda_{4} \Phi_{1}^{\dagger} \Phi_{2} \Phi_{2}^{\dagger} \Phi_{1}+\frac{\lambda_{5}}{2}\left(\left(\Phi_{1}^{\dagger} \Phi_{2}\right)^{2}+\text { h.c. }\right) \\
& +\frac{1}{2} m_{S}^{2} \Phi_{S}^{2}+\frac{\lambda_{6}}{8} \Phi_{S}^{4}+\lambda_{7}\left(\Phi_{1}^{\dagger} \Phi_{1}\right) \Phi_{S}^{2}+\lambda_{8}\left(\Phi_{2}^{\dagger} \Phi_{2}\right) \Phi_{S}^{2}
\end{aligned}
$$

where all parameters are real due to CP-conservation. After electroweak symmetry breaking the two Higgs doublets and the real singlet acquire VEVs $\omega_{i} \in \mathbb{R}$. As a first analysis of the N2HDM vacuum structure performed in ref. [64] has shown, the N2HDM exhibits a different vacuum structure than the $2 \mathrm{HDM}$. The impact of the N2HDM vacuum structure has been further studied in [71] by applying the method of ref. [72]. Additionally, loop corrections and finite temperature effects may change the vacuum texture, so that we expand the doublet and singlet fields including the most general vacuum structure, as

$$
\Phi_{1}=\frac{1}{\sqrt{2}}\left(\begin{array}{c}
\rho_{1}+\mathrm{i} \eta_{1} \\
\zeta_{1}+\omega_{1}+\mathrm{i} \psi_{1}
\end{array}\right), \quad \Phi_{2}=\frac{1}{\sqrt{2}}\left(\begin{array}{c}
\rho_{2}+\omega_{\mathrm{CB}}+\mathrm{i} \eta_{2} \\
\zeta_{2}+\omega_{2}+\mathrm{i}\left(\psi_{2}+\omega_{\mathrm{CP}}\right)
\end{array}\right), \quad \Phi_{S}=\zeta_{3}+\omega_{S},
$$

where we have expanded the Higgs fields in terms of the charged field components $\rho_{i}$ and $\eta_{i}(i=1,2)$, and the neutral $\mathrm{CP}$-even and CP-odd fields $\zeta_{i}$ and $\psi_{i}$, respectively. As mentioned above, already the tree-level zero-temperature vacuum can exhibit a quite general structure as compared to the 2HDM. For simplicity we choose for our present vacuum at zero temperature a vacuum structure with the same properties as in the C2HDM, given by

$$
\left.\left\langle\Phi_{1}\right\rangle\right|_{T=0}=\frac{1}{\sqrt{2}}\left(\begin{array}{c}
0 \\
v_{1}
\end{array}\right),\left.\quad\left\langle\Phi_{2}\right\rangle\right|_{T=0}=\frac{1}{\sqrt{2}}\left(\begin{array}{c}
0 \\
v_{2}
\end{array}\right),\left.\quad\langle S\rangle\right|_{T=0}=v_{S},
$$

with the zero temperature VEVs

$$
\left.v_{i} \equiv \omega_{i}\right|_{T=0}, \quad i=1,2, S, \mathrm{CP}, \mathrm{CB} .
$$


The CP-violating and CB VEVs are hence chosen to be vanishing at zero temperature

$$
v_{\mathrm{CP}}=v_{\mathrm{CB}}=0
$$

And the electroweak VEVs $v_{1,2}$ are related to the SM VEV $v \approx 246 \mathrm{GeV}$ by

$$
v_{1}^{2}+v_{2}^{2} \equiv v^{2} .
$$

As in the (C)2HDM, the ratio of the electroweak VEVs $v_{1}$ and $v_{2}$ is defined by the angle $\beta$,

$$
t_{\beta}=\tan \beta \equiv \frac{v_{2}}{v_{1}} .
$$

Requiring the tree-level potential in eq. (2.20) to be minimized at the electroweak vacuum eq. (2.22) yields

$$
\begin{aligned}
v_{2} m_{12}^{2}-v_{1} m_{11}^{2} & =\frac{v_{1}}{2}\left(v_{1}^{2} \lambda_{1}+v_{2}^{2} \lambda_{345}+v_{S}^{2} \lambda_{7}\right) \\
v_{1} m_{12}^{2}-v_{2} m_{22}^{2} & =\frac{v_{2}}{2}\left(v_{1}^{2} \lambda_{345}+v_{2}^{2} \lambda_{2}+v_{S}^{2} \lambda_{8}\right) \\
-m_{S}^{2} v_{s} & =\frac{v_{s}}{2}\left(v_{1}^{2} \lambda_{7}+v_{2}^{2} \lambda_{8}+v_{S}^{2} \lambda_{6}\right)
\end{aligned}
$$

with

$$
\lambda_{345} \equiv \lambda_{3}+\lambda_{4}+\lambda_{5}
$$

Equation (2.27) allows to trade the parameters $m_{11}^{2}, m_{22}^{2}$ and $m_{S}^{2}$ for the VEVs $v_{1,2, S}$ resulting in the mass matrix for the CP-even neutral fields $\zeta_{1,2,3}$ at zero temperature

$$
M_{\text {Scalar }}^{2}=\left(\begin{array}{ccc}
v^{2} \lambda_{1} c_{\beta}^{2}+m_{12}^{2} t_{\beta} & v^{2} \lambda_{345} c_{\beta} s_{\beta}-m_{12}^{2} & v v_{S} \lambda_{7} c_{\beta} \\
v^{2} \lambda_{345} c_{\beta} s_{\beta}-m_{12}^{2} & v^{2} \lambda_{2} s_{\beta}^{2}+m_{12}^{2} / t_{\beta} & v v_{S} \lambda_{8} s_{\beta} \\
v v_{S} \lambda_{7} c_{\beta} & v v_{S} \lambda_{8} s_{\beta} & v_{S}^{2} \lambda_{6}
\end{array}\right)
$$

The matrix $R$ rotates the mass matrix $M_{\text {Scalar }}^{2}$ in the mass eigenstates $H_{1,2,3}$

$$
\left(\begin{array}{l}
H_{1} \\
H_{2} \\
H_{3}
\end{array}\right)=R\left(\begin{array}{c}
\rho_{1} \\
\rho_{2} \\
\rho_{S}
\end{array}\right),
$$

with the mass eigenvalues

$$
R M_{\text {Scalar }}^{2} R^{T}=\operatorname{diag}\left(m_{H_{1}}^{2}, m_{H_{2}}^{2}, m_{H_{3}}^{2}\right) .
$$

We use the same convention as in the C2HDM where the mass eigenstates are ordered by ascending masses as

$$
m_{H_{1}} \leq m_{H_{2}} \leq m_{H_{3}} .
$$

The N2HDM has 12 real independent parameters where we chose as many parameters as possible with a physical meaning. We take eq. (2.27) to trade the potential parameters $m_{11}^{2}$, $m_{22}^{2}$ and $m_{S}^{2}$ for the SM and singlet VEVs $v$ and $v_{S}$, and $\tan \beta$. Moreover, we express the 
quartic couplings $\lambda_{i}$ in terms of the physical masses and mixing angles. The $\mathbb{Z}_{2}$ breaking mass term $m_{12}^{2}$ is kept as independent parameter. This yields the following set of input parameters

$$
\alpha_{1}, \quad \alpha_{2}, \quad \alpha_{3}, \quad t_{\beta}, \quad v, \quad v_{S}, m_{H_{1,2,3}}, \quad m_{A}, m_{H^{ \pm}}, m_{12}^{2} .
$$

To avoid FCNC in the N2HDM the same types as in the C2HDM, given in table 1, can be used since the singlet field $\Phi_{S}$ does not couple to fermions. For more details and a phenomenological discussion of the N2HDM we refer to [64].

\subsection{One-loop effective potential at finite temperature}

In the following we briefly repeat the main ingredients for the one-loop effective potential at non-zero temperature. For details, we refer to $[45,60,73]$ which can be generalized to the N2HDM.

The loop-corrected effective potential splits into the tree-level potential $V_{\text {tree }}$, the oneloop correction at zero temperature given by the Coleman-Weinberg $(\mathrm{CW})$ potential $V_{\mathrm{CW}}$, and the temperature-dependent part $V_{T}$, so that it reads

$$
V \equiv V_{\text {tree }}+V_{\mathrm{CW}}+V_{T}
$$

where $V_{\text {tree }}$ is given by eq. (2.1) for the C2HDM and eq. (2.20) for the N2HDM, after replacing the doublet fields of the $\mathrm{C} 2 \mathrm{HDM}$ with their classical constant field configuration given in eqs. (2.3), (2.4), and the doublet and singlet fields of the N2HDM with eq. (2.22). The CW potential [74] in the $\overline{\mathrm{MS}}$ scheme is given by

$$
V_{\mathrm{CW}}=\sum_{j} \frac{n_{j}}{64 \pi^{2}}(-1)^{2 s_{i}} m_{j}^{4}\left[\ln \left(\frac{m_{j}^{2}}{\mu^{2}}\right)-c_{j}\right],
$$

where $n_{j}$ are the degrees of freedom, $s_{j}$ the spin and $m_{j}$ the mass of the specific particle $j$. The sum extends over the Higgs and Goldstone bosons, the massive gauge bosons, the photon and the fermions, so that both for the C2HDM and the N2HDM we have

$$
j \in\left\{H_{1}, H_{2}, H_{3}, A, H^{ \pm}, G^{0}, G^{ \pm}, W^{ \pm}, Z, \gamma, f\right\} .
$$

The mass $m_{j}$ is the mass eigenvalue of particle $j$ obtained from the tree-level mass matrix expressed in terms of the general VEV configuration $\omega_{i}$, with $i=1,2, \mathrm{CP}, \mathrm{CB}$ for the $\mathrm{C} 2 \mathrm{HDM}$ and $i=1,2, S, \mathrm{CP}, \mathrm{CB}$ for the N2HDM. Applying the Landau gauge in the calculation of the CW potential allows us to drop the ghost contributions in the analysis, but we need to account for the possibility that the Goldstone bosons get massive. The Goldstone bosons as well the photon are massless at $T=0$, but for field configurations different from the tree-level VEVs at $T=0$, which is needed for the minimization procedure, they can acquire an effective mass term. Furthermore, we allow for unphysical vacuum structures with non-zero $\omega_{\mathrm{CB}}$ inducing additionally unphysical masses. We also account for the possibility of the generation of a CP-violating VEV $\omega_{\mathrm{CP}}$. For the neutral 
scalars $\Phi^{0}=H_{1}, H_{2}, H_{3}, A, G^{0}$, the charged scalars $\Phi^{ \pm}=H^{+}, H^{-}, G^{+}, G^{-}$, the leptons $l^{+}, l^{-}$, quarks $q, \bar{q}$ and longitudinal and transversal gauge bosons $V_{L}=Z_{L}, W_{L}^{+}, W_{L}^{-}, \gamma_{L}$ and $V_{T}=Z_{T}, W_{T}^{+}, W_{T}^{-}, \gamma_{T}$ the degrees of freedom in eq. (2.35) read

$$
\begin{aligned}
& n_{\Phi^{0}}=1, \quad n_{\Phi^{ \pm}}=2, \quad n_{V_{T}}=2, \quad n_{V_{L}}=1, \\
& n_{l^{+}}=2, \quad n_{l^{-}}=2, \quad n_{q}=6, \quad n_{\bar{q}}=6 \text {. }
\end{aligned}
$$

The renormalisation scale is chosen as $\mu=v \approx 246.22 \mathrm{GeV}$ and the $\overline{\mathrm{MS}}$ renormalisation constants read

$$
c_{j}=\left\{\begin{array}{ll}
\frac{5}{6}, & j=W^{ \pm}, Z, \gamma \\
\frac{3}{2}, & \text { otherwise }
\end{array} .\right.
$$

The thermal corrections $V_{T}$ comprise the daisy resummation [75] of the $n=0$ Matsubara modes of the longitudinal components of the gauge bosons $W_{L}^{ \pm}, Z_{L}, \gamma_{L}$ and the bosons $\Phi^{0}, \Phi^{ \pm}$. This Debye correction adds to their masses at zero temperature. The thermal corrections $V_{T}$ can be cast in the form [76, 77]

$$
V_{T}=\sum_{k} n_{k} \frac{T^{4}}{2 \pi^{2}} J_{ \pm}^{(k)} .
$$

The sum extends over $k=W_{T, L}^{ \pm}, Z_{L, T}, \gamma_{L, T}, \Phi^{0}, \Phi^{ \pm}, l^{ \pm}, q, \bar{q}$. Denoting the mass eigenvalues including the thermal corrections for the particles $k$ by $\overline{m_{k}}$, we have for $J_{ \pm}$(cf. e.g. [78] $)^{1}$

$$
J_{ \pm}^{(k)}= \begin{cases}J_{-}\left(\frac{m_{k}^{2}}{T^{2}}\right)-\frac{\pi}{6}\left(\bar{m}_{k}^{3} / T^{3}-m_{k}^{3} / T^{3}\right), & k=W_{L}, Z_{L}, \gamma_{L}, \Phi^{0}, \Phi^{ \pm} \\ J_{-}\left(\frac{m_{k}^{2}}{T^{2}}\right) & k=W_{T}, Z_{T}, \gamma_{T} \\ J_{+}\left(\frac{m_{k}^{2}}{T^{2}}\right) & k=\text { fermion }\end{cases}
$$

with the thermal integrals

$$
J_{ \pm}(x)=\mp \int_{0}^{\infty} d x x^{2} \log \left[1 \pm \exp \left(-\sqrt{x^{2}+m_{k}^{2} / T^{2}}\right)\right]
$$

where $J_{+}\left(J_{-}\right)$applies for $k$ representing a fermion (boson). The general formulae for the thermal masses can be found in [73]. For the numerical evaluation of the effective potential at finite temperatures and the further minimization procedure we use the code BSMPT v1.1.2 [73] and we refer to [73] for a more detailed discussion of the used numerical approximations in the thermal integrals in eq. (2.39).

\footnotetext{
${ }^{1}$ We use the 'Arnold-Espinosa' [79, 80] approach for the inclusion of the Debye corrected masses. For further remarks on the approach and how it compares to the 'Parwani' approach, see [79, 80]. Further related discussion as well as approaches using three-dimensional effective field theories based on high-temperature dimensional reduction can be found in [51, 81-95].
} 


\section{Renormalisation}

At one-loop level the masses and mixing angles differ from those extracted from the treelevel potential. In order to take into account the one-loop effects and at the same time enable an efficient scan in the parameter space of the model, we renormalize the loopcorrected masses and mixing angles such that they are equal to their tree-level values. This allows us to use them directly as input values for our scan. The scheme has been introduced in ref. [45] where it was applied to the 2HDM. In [60] it was extended to the C2HDM. We therefore show here only the procedure for the N2HDM. The renormalised loop-corrected potential $\hat{V}$ is obtained by adding the counterterm potential $V_{\mathrm{CT}}$

$$
\hat{V}=V+V_{\mathrm{CT}}=V_{\text {tree }}+V_{\mathrm{CW}}+V_{T}+V_{\mathrm{CT}}
$$

with the counterterm potential given by

$$
V_{\mathrm{CT}}=\sum_{i=1} \frac{\partial V_{\text {tree }}}{\partial p_{i}} \delta p_{i}+\sum_{k=1}\left(\phi_{k}+\omega_{k}\right) \delta T_{k}
$$

where $p_{i}$ stands for the parameters of the tree-level potential. Furthermore, for each field direction $\phi_{k}$ which is allowed to develop a VEV, a tadpole counterterm $\delta T_{k}$ is introduced. For the N2HDM these are $\delta T_{1}, \delta T_{2}, \delta T_{S}, \delta T_{\mathrm{CP}}$ and $\delta T_{\mathrm{CB}}$. The renormalisation conditions for the scheme described above read

$$
\left.\partial_{\phi_{i}} V_{\mathrm{CT}}(\phi)\right|_{\langle\phi\rangle_{T=0}}=-\left.\partial_{\phi_{i}} V_{\mathrm{CW}}(\phi)\right|_{\langle\phi\rangle_{T=0}}
$$

and

$$
\left.\partial_{\phi_{i}} \partial_{\phi_{j}} V_{\mathrm{CT}}(\phi)\right|_{\langle\phi\rangle_{T=0}}=-\left.\partial_{\phi_{i}} \partial_{\phi_{j}} V_{\mathrm{CW}}(\phi)\right|_{\langle\phi\rangle_{T=0}},
$$

where $\langle\phi\rangle_{T=0}$ denotes the tree-level vacuum state at zero temperature. The conditions eq. (3.3) and eq. (3.4) ensure that at zero temperature the tree-level minimum remains a local minimum at one-loop level. We check numerically if it also the global one. Additionally, the second set of conditions in eq. (3.4) ensures that the masses and mixing angles derived from the loop-corrected effective potential remain at their tree-level values. In general, the renormalisation conditions result in an overconstrained system of equations which can be solved by imposing additional assumptions. In the case of the N2HDM eqs. (3.3) and (3.4) lead to a two-dimensional solution space which can be fixed by imposing

$$
\delta \lambda_{4}=0 \quad \text { and } \quad \delta T_{S}=0 .
$$


Solving then for the counterterm parameters yields

$$
\begin{aligned}
& \delta m_{11}^{2}=\frac{1}{2}\left[\frac{v_{s}}{v_{1}} H_{\zeta_{1}, \zeta_{S}}^{\mathrm{CW}}+\frac{v_{2}}{v_{1}}\left(H_{\zeta_{1}, \zeta_{2}}^{\mathrm{CW}}-H_{\eta_{1}, \eta_{2}}^{\mathrm{CW}}\right)+2 H_{\eta_{1}, \eta_{1}}^{\mathrm{CW}}-5 H_{\eta_{1}, \eta_{1}}^{\mathrm{CW}}+H_{\zeta_{1}, \zeta_{1}}^{\mathrm{CW}}\right] \\
& \delta m_{22}^{2}=\frac{1}{2}\left[\frac{v_{s}}{v_{2}} H_{\zeta_{2}, \zeta_{S}}^{\mathrm{CW}}+H_{\zeta_{2}, \zeta_{2}}^{\mathrm{CW}}-3 H_{\eta_{2}, \eta_{2}}^{\mathrm{CW}}+\frac{v_{1}}{v_{2}}\left(H_{\zeta_{1}, \zeta_{2}}^{\mathrm{CW}}-H_{\eta_{1}, \eta_{2}}^{\mathrm{CW}}\right)+5 \frac{v_{1}^{2}}{v_{2}^{2}}\left(H_{\eta_{1}, \eta_{1}}^{\mathrm{CW}}-H_{\eta_{1}, \eta_{1}}^{\mathrm{CW}}\right)\right] \\
& \delta m_{12}^{2}=H_{\eta_{1}, \eta_{2}}^{\mathrm{CW}}+\frac{v_{1}}{v_{2}}\left(H_{\eta_{1}, \eta_{1}}^{\mathrm{CW}}-H_{\eta_{1}, \eta_{1}}^{\mathrm{CW}}\right) \\
& \delta \lambda_{1}=\frac{1}{v_{1}^{2}}\left(2 H_{\eta_{1}, \eta_{1}}^{\mathrm{CW}}-H_{\eta_{1}, \eta_{1}}^{\mathrm{CW}}-H_{\zeta_{1}, \zeta_{1}}^{\mathrm{CW}}\right) \\
& \delta \lambda_{2}=\frac{1}{v_{2}^{2}}\left(H_{\eta_{2}, \eta_{2}}^{\mathrm{CW}}-H_{\zeta_{2}, \zeta_{2}}^{\mathrm{CW}}\right)+2 \frac{v_{1}^{2}}{v_{2}^{4}}\left(H_{\eta_{1}, \eta_{1}}^{\mathrm{CW}}-H_{\eta_{1}, \eta_{1}}^{\mathrm{CW}}\right) \\
& \delta \lambda_{3}=\frac{1}{v_{2}^{2}}\left(H_{\eta_{1}, \eta_{1}}^{\mathrm{CW}}-H_{\eta_{1}, \eta_{1}}^{\mathrm{CW}}\right)+\frac{1}{v_{1} v_{2}}\left(H_{\eta_{1}, \eta_{2}}^{\mathrm{CW}}-H_{\zeta_{1}, \zeta_{2}}^{\mathrm{CW}}\right) \\
& \delta \lambda_{4}=0 \\
& \delta \lambda_{5}=\frac{2}{v_{2}^{2}}\left(H_{\psi_{1}, \psi_{1}}^{\mathrm{CW}}-2 H_{\eta_{1}, \eta_{1}}^{\mathrm{CW}}\right) \\
& \delta m_{S}^{2}=\frac{1}{2}\left(H_{\zeta_{S}, \zeta_{S}}^{\mathrm{CW}}+\frac{v_{2}}{v_{s}} H_{\zeta_{2}, \zeta_{S}}^{\mathrm{CW}}+\frac{v_{1}}{v_{s}} H_{\zeta_{1}, \zeta_{S}}^{\mathrm{CW}}-\frac{3}{v_{S}} N_{\zeta_{S}}^{\mathrm{CW}}\right) \\
& \delta \lambda_{6}=\frac{1}{v_{s}^{3}}\left(N_{\zeta_{S}}^{\mathrm{CW}}-v_{s} H_{\zeta_{S}, \zeta_{S}}^{\mathrm{CW}}\right) \\
& \delta \lambda_{7}=-\frac{1}{v_{s} v_{1}} H_{\zeta_{1}, \zeta_{S}}^{\mathrm{CW}} \\
& \delta \lambda_{8}=-\frac{1}{v_{s} v_{2}} H_{\zeta_{2}, \zeta_{S}}^{\mathrm{CW}} \\
& \delta T_{1}=H_{\eta_{1}, \eta_{1}}^{\mathrm{CW}} v_{1}+H_{\eta_{1}, \eta_{2}}^{\mathrm{CW}} v_{2}-N_{\zeta_{1}}^{\mathrm{CW}} \\
& \delta T_{2}=\frac{v_{1}^{2}}{v_{2}}\left(H_{\eta_{1}, \eta_{1}}^{\mathrm{CW}}-H_{\psi_{1}, \psi_{1}}^{\mathrm{CW}}\right)+H_{\eta_{1}, \eta_{2}}^{\mathrm{CW}} v_{1}+H_{\eta_{2}, \eta_{2}} v_{2}-N_{\rho_{2}}^{\mathrm{CW}} \\
& \delta T_{S}=0 \\
& \delta T_{3}=\frac{v_{1}^{2}}{v_{2}} H_{\zeta_{1}, \psi_{1}}^{\mathrm{CW}}+H_{\zeta_{1}, \psi_{2}}^{\mathrm{CW}} v_{1}-N_{\psi_{2}}^{\mathrm{CW}} \\
& \delta T_{C B}=-N_{\rho_{2}}^{\mathrm{CW}} \text {, }
\end{aligned}
$$

with the shorthand notations

$$
N_{\phi_{i}}^{\mathrm{CW}} \equiv \partial_{\phi_{i}} V_{\mathrm{CW}}
$$

and

$$
H_{\phi_{i} \phi_{j}}^{\mathrm{CW}}=\partial_{\phi_{i}} \partial_{\phi_{j}} V_{\mathrm{CW}} .
$$

In ref. [96] formulae for both the first and the second derivatives of the CW potential have been derived in the Landau gauge basis. 


\section{Numerical analysis}

\subsection{Minimisation of the effective potential}

The EWPT is of strong first order if the baryon-washout condition is met which requires that the ratio $\xi_{c}$ of the critical VEV $v_{c},{ }^{2}$ and the critical temperature $T_{c}$ is larger than one $[11,77,102]$,

$$
\xi_{c} \equiv \frac{v_{c}}{T_{c}} \geq 1 .
$$

The VEV $v$ at the temperature $T$ is given by

$$
v(T)=\sqrt{\omega_{1}^{2}(T)+\omega_{2}^{2}(T)+\omega_{\mathrm{CP}}^{2}(T)+\omega_{\mathrm{CB}}^{2}(T)},
$$

where the $\omega_{i}$ are the field configurations that minimise the loop-corrected effective potential at finite temperature $T$. Note that we do not include the singlet VEV $\omega_{S}$ (present in the $\mathrm{N} 2 \mathrm{HDM}$ ) in eq. (4.2), but we take $\omega_{S}$ into account for the minimisation procedure. Since the electroweak sphaleron couples only to particles charged under $\mathrm{SU}(2)_{L}$, the singlet VEV can be dropped in the calculation of the critical VEV in eq. (4.2). The critical temperature $T_{c}$ is defined as the temperature where the potential has developed two degenerate minima. In order to compute the global electroweak minimum of the one-loop corrected effective C2HDM and N2HDM potentials of eq. (3.1) we implemented both models in BSMPT v1.1.2 [73] which also calculates the strength $\xi_{c}$ of the phase transition.

\subsection{Constraints and parameter scan}

For simplicity, in the numerical analysis we discuss only Type I and II of the models. The parameter samples used for the numerical investigation in this paper have to satisfy theoretical and experimental constraints. We obtained them by performing scans in the parameter spaces of the C2HDM and N2HDM, respectively. For the scans we required that one of the neutral Higgs bosons, denoted by $h$ in the following, behaves SM-like and has a mass of $m_{h}=125.09 \mathrm{GeV}[26]$. The scan ranges for all the input parameters are given in table 2 for Type I (T1) and in table 3 for Type II (T2) of the N2HDM, for the C2HDM they are given in table 4 for Type I and in table 5 for Type II. We introduce here the notation $m_{H_{\downarrow} / H_{\uparrow}}$ for the masses of the lighter/heavier of the two non-SM-like neutral Higgs bosons.

As for the SM parameters of our analysis, we use the fine structure constant taken at the $Z$ boson mass scale [103, 104],

$$
\alpha_{\mathrm{EM}}^{-1}\left(M_{Z}^{2}\right)=128.962,
$$

and the masses for the massive gauge bosons are chosen as

$$
m_{W}=80.385 \mathrm{GeV} \quad \text { and } \quad m_{Z}=91.1876 \mathrm{GeV} .
$$

\footnotetext{
${ }^{2}$ We remind the reader that while the value of the effective potential at the minimum is gauge independent, this is not the case for the value of the VEV. The issue of gauge dependence has been analysed in the literature [46, 97-101]. Gauge-invariant approaches have been proposed within simpler models applying certain approximations. While a gauge-invariant treatment for the analysis of the EWPT would certainly be preferred, this is beyond the scope of the analysis for the model investigated in this paper.
} 


\begin{tabular}{|cccccc|}
\hline$m_{h}$ & $m_{H_{\downarrow}}$ & $\begin{array}{c}m_{H_{\uparrow}} \\
\text { in } \mathrm{GeV}\end{array}$ & $m_{A}$ & $m_{H^{ \pm}}$ & $\begin{array}{c}m_{12}^{2} \\
\text { in } \mathrm{GeV}^{2}\end{array}$ \\
\hline 125.09 & {$[30,1500]$} & {$[30,1500]$} & {$[30,1500]$} & {$[30,1500]$} & {$\left[10^{-3}, 10^{5}\right]$} \\
\hline$\alpha_{1}$ & $\alpha_{2}$ & $\alpha_{3}$ & $\tan \beta$ & $v_{S}[\mathrm{GeV}]$ & \\
\hline$\left[-\frac{\pi}{2}, \frac{\pi}{2}\right)$ & {$\left[-\frac{\pi}{2}, \frac{\pi}{2}\right)$} & {$\left[-\frac{\pi}{2}, \frac{\pi}{2}\right)$} & {$[0.8,20]$} & {$[1,3000]$} & \\
\hline
\end{tabular}

Table 2. Parameter ranges for the N2HDM T1 input parameters used in ScannerS.

\begin{tabular}{|cccccc|}
\hline$m_{h}$ & $m_{H_{\downarrow}}$ & $\begin{array}{c}m_{H_{\uparrow}} \\
\text { in } \mathrm{GeV}\end{array}$ & $m_{A}$ & $m_{H^{ \pm}}$ & $\begin{array}{c}m_{12}^{2} \\
\text { in } \mathrm{GeV}^{2}\end{array}$ \\
\hline 125.09 & {$[30,1500]$} & {$[30,1500]$} & {$[30,1500]$} & {$[580,1500]$} & {$\left[10^{-3}, 10^{5}\right]$} \\
\hline$\alpha_{1}$ & $\alpha_{2}$ & $\alpha_{3}$ & $\tan \beta$ & $v_{S}[\mathrm{GeV}]$ & \\
\hline$\left[-\frac{\pi}{2}, \frac{\pi}{2}\right)$ & {$\left[-\frac{\pi}{2}, \frac{\pi}{2}\right)$} & {$\left[-\frac{\pi}{2}, \frac{\pi}{2}\right)$} & {$[0.8,20]$} & {$[1,3000]$} & \\
\hline
\end{tabular}

Table 3. Parameter ranges for the N2HDM T2 input parameters used in ScannerS.

\begin{tabular}{|ccccc|}
\hline$m_{h}$ & $m_{H_{\downarrow}}$ & $\begin{array}{c}m_{H_{\uparrow}} \\
\text { in } \mathrm{GeV}\end{array}$ & $m_{H^{ \pm}}$ & $\begin{array}{c}\operatorname{Re} m_{12}^{2} \\
\text { in } \mathrm{GeV}^{2}\end{array}$ \\
\hline 125.09 & {$[30,1500]$} & {$[30,1500]$} & {$[30,1500]$} & {$\left[10^{-3}, 10^{5}\right]$} \\
\hline$\alpha_{1}$ & $\alpha_{2}$ & $\alpha_{3}$ & $\tan \beta$ & \\
\hline$\left[-\frac{\pi}{2}, \frac{\pi}{2}\right)$ & {$\left[-\frac{\pi}{2}, \frac{\pi}{2}\right)$} & {$\left[-\frac{\pi}{2}, \frac{\pi}{2}\right)$} & {$[0.8,20]$} & \\
\hline
\end{tabular}

Table 4. Parameter ranges for the C2HDM T1 input parameters used in ScannerS.

\begin{tabular}{|ccccc|}
\hline$m_{h}$ & $m_{H_{\downarrow}}$ & $\begin{array}{c}m_{H_{\uparrow}} \\
\text { in } \mathrm{GeV}\end{array}$ & $m_{H^{ \pm}}$ & $\begin{array}{c}\text { Re } m_{12}^{2} \\
\text { in } \mathrm{GeV}^{2}\end{array}$ \\
\hline 125.09 & {$[30,1500]$} & {$[30,1500]$} & {$[580,1500]$} & {$\left[10^{-3}, 10^{5}\right]$} \\
\hline$\alpha_{1}$ & $\alpha_{2}$ & $\alpha_{3}$ & $\tan \beta$ & \\
\hline$\left[-\frac{\pi}{2}, \frac{\pi}{2}\right)$ & {$\left[-\frac{\pi}{2}, \frac{\pi}{2}\right)$} & {$\left[-\frac{\pi}{2}, \frac{\pi}{2}\right)$} & {$[0.8,20]$} & \\
\hline
\end{tabular}

Table 5. Parameter ranges for the C2HDM T2 input parameters used in ScannerS.

The lepton masses are set to

$$
m_{e}=0.511 \mathrm{MeV}, \quad m_{\mu}=105.658 \mathrm{MeV}, \quad m_{\tau}=1.777 \mathrm{GeV},
$$

and the light quark masses to

$$
m_{u}=m_{d}=m_{s}=100 \mathrm{MeV} .
$$


To be consistent with the CMS and ATLAS analyses, we take the on-shell top quark mass as $[105]$

$$
m_{t}=172.5 \mathrm{GeV}
$$

and the recommended charm and bottom quark on-shell masses

$$
m_{c}=1.51 \mathrm{GeV} \text { and } m_{b}=4.92 \mathrm{GeV} \text {. }
$$

The CKM matrix is taken to be real, with the CKM matrix elements given by [103]

$$
V_{\mathrm{CKM}}=\left(\begin{array}{ccc}
V_{u d} & V_{u s} & V_{u b} \\
V_{c d} & V_{c s} & V_{c b} \\
V_{t d} & V_{t s} & V_{t b}
\end{array}\right)=\left(\begin{array}{ccc}
0.97427 & 0.22536 & 0.00355 \\
-0.22522 & 0.97343 & 0.0414 \\
0.00886 & -0.0405 & 0.99914
\end{array}\right)
$$

Finally, the electroweak VEV is set to

$$
v=1 / \sqrt{\sqrt{2} G_{F}}=246.22 \mathrm{GeV} .
$$

To be consistent with recent flavour constraints, we test for the compatibility with $\mathcal{R}_{b}[106,107]$ and $B \rightarrow X_{s} \gamma[107-111]$ in the $m_{H^{ \pm}}-\tan \beta$ plane. For T2, this implies that the charged Higgs mass has to be above $580 \mathrm{GeV}$ [111] whereas in $\mathrm{T} 1$ this bound is much weaker and is strongly correlated with $\tan \beta .^{3}$ Compatibility with the electroweak precision data is checked through the oblique parameters $S, T$ and $U$ [112] where we apply the general procedure for extended Higgs sectors given in [113, 114]. Including the full correlations, we demand $2 \sigma$ compatibility with the SM fit [115].

In order to avoid degenerate Higgs signals we impose a mass window between the nonSM- and SM-like Higgs bosons so that masses $\left|m_{H_{i} \neq h}-m_{h}\right|<5 \mathrm{GeV}$ are excluded from the analysis. We use the program ScannerS [64, 116-118] to search for valid parameter points. The program allows to check for boundedness from below of the tree-level potential and, for the C2HDM, uses the tree-level discriminant of [119] to ensure the electroweak vacuum to be the global minimum at tree level. For the N2HDM, all tree-level minima have been implemented in ScannerS and are compared numerically to find the global minimum. To check for consistency with the Higgs exclusion limits from LEP, Tevatron and LHC HiggsBounds5 .5.0 [120-122] is used, and the SM-like Higgs rates are required to be within the $2 \sigma$ range of the SM which is checked by HiggsSignals2.3.0 [123]. The required decay widths and branching ratios are obtained from C2HDM_HDECAY [65] for the C2HDM and from N2HDECAY $[64,124]$ for the N2HDM. Both codes are based on the implementation of the C2HDM and the N2HDM each in the existing code HDECAY $[125,126]$. Due to the CP violation, in the C2HDM also compatibility with the electric dipole moments [127] has to be checked.The most stringent limit is provided by the ACME collaboration [128]. Moreover, we take into account for both models the impact of the recent di-Higgs searches in the final states $4 b[129,130],(2 b)(2 \tau)[131,132]$ and $(2 b)(2 \gamma)[133]$ on the viable parameter space. These searches are also implemented in HiggsBounds and HiggsSignals.

\footnotetext{
${ }^{3}$ Many of the experimental constraints applied on the $2 \mathrm{HDM}$ also hold for the N2HDM, since these constraints are only sensitive to the charged Higgs boson so that the calculation of the 2HDM can be taken over to the N2HDM [64]. In this way we are able to use the same constraints for the C2HDM and N2HDM.
} 
In addition to these checks, we impose the requirement that the tree-level minimum of the potential is still the global electroweak minimum at one-loop level. The one-loop minimum is determined by numerical minimisation of the one-loop potential at zero temperature and checked against the tree-level value. In the following, all parameter points providing an SFOEWPT also have an NLO stable global minimum at zero temperature. Furthermore, we demand an approximated NLO unitarity. The tree-level perturbative unitarity relations for the N2HDM read $[64,73]$

$$
\left|\frac{1}{2} a_{1,2,3}, b_{ \pm}, c_{ \pm}, e_{1,2}, f_{ \pm}, f_{1}, p_{1}, s_{1}, s_{2}\right|<8 \pi,
$$

with

$$
\begin{aligned}
b_{ \pm} & =\frac{1}{2}\left(\lambda_{1}+\lambda_{2}\right) \pm \frac{1}{2} \sqrt{\left(\lambda_{1}-\lambda_{2}\right)^{2}+4 \lambda_{4}^{2}}, \\
c_{ \pm} & =\frac{1}{2}\left(\lambda_{1}+\lambda_{2}\right) \pm \frac{1}{2} \sqrt{\left(\lambda_{1}-\lambda_{2}\right)^{2}+4 \lambda_{5}^{2}}, \\
e_{1} & =\lambda_{3}+2 \lambda_{4}-3 \lambda_{5}, \\
e_{2} & =\lambda_{3}-\lambda_{5} \\
f_{+} & =\lambda_{3}+2 \lambda_{4}+3 \lambda_{5}, \\
f_{-} & =\lambda_{3}+\lambda_{5}, \\
f_{1} & =\lambda_{3}+\lambda_{4}, \\
p_{1} & =\lambda_{3}-\lambda_{4}, \\
s_{1} & =\lambda_{7}, \\
s_{2} & =\lambda_{8}
\end{aligned}
$$

and the eigenvalues $a_{1,2,3}$ that are the real roots of the cubic polynomial

$$
\begin{aligned}
f(x)= & x^{3}+x^{2}\left[-6\left(\lambda_{1}+\lambda_{2}\right)-3 \lambda_{6}\right] \\
& +x\left[36 \lambda_{1} \lambda_{2}-16 \lambda_{3}^{2}-16 \lambda_{3} \lambda_{4}-4 \lambda_{4}^{2}+18 \lambda_{1} \lambda_{6}+18 \lambda_{2} \lambda_{6}-4 \lambda_{7}^{2}-4 \lambda_{8}^{2}\right] \\
& +4\left(-27 \lambda_{1} \lambda_{2} \lambda_{6}+12 \lambda_{3}^{2} \lambda_{6}+12 \lambda_{3} \lambda_{4} \lambda_{6}+3 \lambda_{4}^{2} \lambda_{6}+6 \lambda_{2} \lambda_{7}^{2}-8 \lambda_{3} \lambda_{7} \lambda_{8}\right. \\
& \left.-4 \lambda_{4} \lambda_{7} \lambda_{8}+6 \lambda_{1} \lambda_{8}^{2}\right) .
\end{aligned}
$$

By replacing $\lambda_{i} \rightarrow \lambda_{i}+\delta \lambda_{i}$ in eq. (4.12), the NLO effects on the unitarity of the $S$-matrix can be approximated and checked in the parameter scan. The counterterms $\delta \lambda_{i}$ in the $\mathrm{N} 2 \mathrm{HDM}$ are given in eq. (3.6) and are calculated numerically during the minimisation procedure. The corresponding relations for the $\mathrm{C} 2 \mathrm{HDM}$ counterterms and perturbative unitarity relations are given in $[31,65,73]$. By imposing these additional constraints at NLO, it can happen that the parameter sample is significantly reduced. In table 6 a list of the remaining parameter sample is given after imposing the NLO constraints and the requirement for an SFOEWPT. These numbers have to be taken with a grain of salt, however, as dedicated scans adapted to specific requirements like e.g. not loosing points due to NLO unitarity, would change this picture. The numbers are simply meant to show that NLO constraints and/or the requirement of an SFOEWPT have an effect on the parameter sample. 


\begin{tabular}{|lcccc|}
\hline Applied constraint & C2HDM (T1) & C2HDM (T2) & N2HDM (T1) & N2HDM (T2) \\
\hline Total number of parameter points & 233163 & 1029538 & 271743 & 302653 \\
\hline NLO vacuum stability & $97.32 \%$ & $90.36 \%$ & $83.64 \%$ & $87.38 \%$ \\
\hline $\begin{array}{l}\text { NLO vacuum stability + NLO } \\
\text { perturbative unitarity }\end{array}$ & $91.00 \%$ & $79.451 \%$ & $80.32 \%$ & $85.96 \%$ \\
\hline $\begin{array}{l}\text { SFOEWPT + NLO vacuum stabil- } \\
\text { ity + NLO perturbative unitarity }\end{array}$ & $0.012 \%$ & $0.003 \%$ & $0.379 \%$ & $0.022 \%$ \\
\hline
\end{tabular}

Table 6. Reduction of the number of parameter points before and after applying NLO vacuum stability, NLO perturbative unitarity and an SFOEWPT.

The Higgs spectra of the C2HDM and N2HDM consist of at least three neutral Higgs bosons (in the N2HDM, we additionally have the pseudoscalar). Denoting the lighter and heavier non-SM-like Higgs bosons with masses $m_{H_{\downarrow}}$ and $m_{H_{\uparrow}}$ by $H_{\downarrow}$ and $H_{\uparrow}$, we can have three different mass configurations that we will refer to in the following as heavy mass hierarchy with the mass hierarchy

$$
m_{h}<m_{H_{\downarrow}}<m_{H_{\uparrow}},
$$

semi-inverted mass hierarchy with

$$
m_{H_{\downarrow}}<m_{h}<m_{H_{\uparrow}},
$$

and inverted mass hierarchy with the hierarchy

$$
m_{H_{\downarrow}}<m_{H_{\uparrow}}<m_{h} .
$$

\section{Results}

In the following analysis we investigate to which extent the viable parameter spaces of the C2HDM and N2HDM are constrained by the additional requirement of a strong first order EWPT. This also allows us to investigate the differences that arise due to $\mathrm{CP}$ violation on the one hand and singlet admixture on the other hand. Since we discussed the implications for the phenomenology of the C2HDM already in detail in ref. [60], we start by providing a rather short update of our analysis of the C2HDM by taking into account the newest results for the Higgs data. Subsequently, we discuss in detail the interplay between a strong first order phase transition and the collider phenomenology of the N2HDM. In particular, we study the impact of $\xi_{c} \geq 1$ on the size of the trilinear Higgs self-couplings and the overall mass spectrum. We will provide benchmark scenarios that highlight the connection between a successful SFOEWPT and collider phenomenology with emphasis on Higgs pair production at the LHC. In the end a comparison between the two models will be drawn and the characteristic differences in their phenomenology with respect to a successful SFOEWPT will be discussed. For our analysis we produced for each model of each type about half a million parameter points that respect the theoretical and experimental constraints listed in section 4.2. These parameter points are checked for a successful SFOEWPT by using the program BSMPT v1.1.2 [73]. 


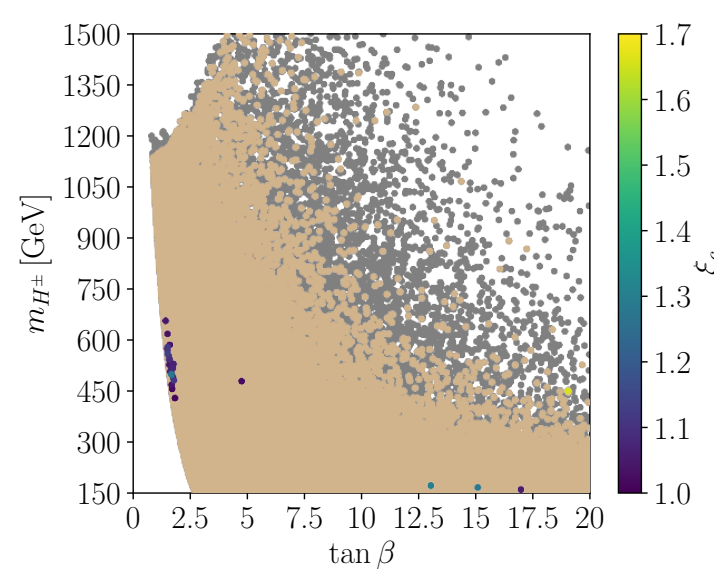

(a)

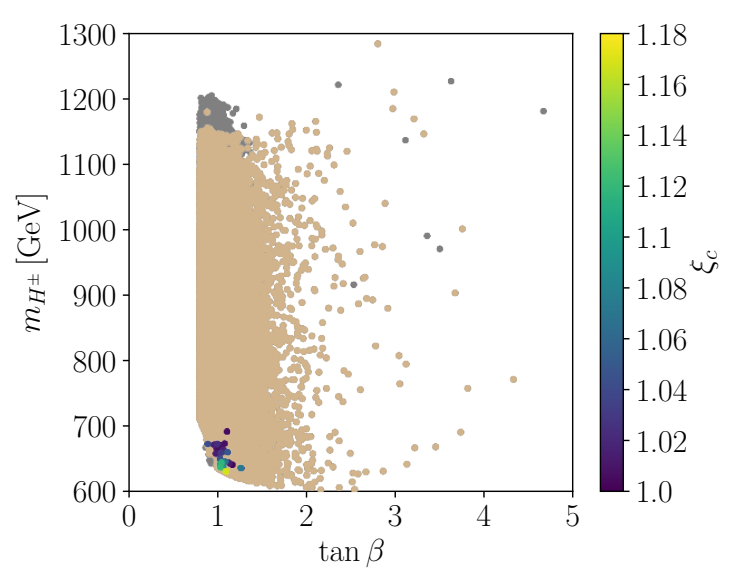

(b)

Figure 1. C2HDM: the mass $m_{H^{ \pm}}$versus $\tan \beta$ for T1 (left) and T2 (right). The grey points are all parameter points passing the experimental and theoretical checks of ScannerS. The brown points additionally fulfill NLO vacuum stability and NLO perturbative unitarity constraints. The color code denotes the strength of the phase transition $\xi_{c}$ for $\xi_{c} \geq 1$. The di-Higgs search constraints are included.

\subsection{C2HDM - update}

We start with an update of our analysis in ref. [60] by taking into account the new collider constraints that have been implemented in HiggsSignals 2.3.0 and HiggsBounds 5.5.0. In addition, we increased the scan ranges of all scalar masses from $1 \mathrm{TeV}$ up to $1.5 \mathrm{TeV}$ with the aim to find new valid parameter points featuring a heavy scalar spectrum that provides an SFOEWPT. The subsequent discussion will show that no additional heavy parameter points were found fulfilling the requirement of an SFOEWPT and compatibility with recent collider constraints.

We start the discussion with figure 1 , where the charged Higgs boson mass $m_{H^{ \pm}}$is shown as a function of $\tan \beta$ for the C2HDM T1 (left) and T2 (right). The grey points are all parameter points compatible with the theoretical and recent experimental constraints as described in section 4.2. The brown points additionally provide an NLO stable vacuum and fulfill NLO perturbative unitarity (see also section 4.2). The color code indicates parameter points with values of $\xi_{c} \geq 1$ and thereby all points with an SFOEWPT. In the C2HDM T1 two distinct possible scenarios for parameter points providing an SFOEWPT can be observed. The first region has charged Higgs boson masses of $\sim 450 \mathrm{GeV}$ up to $\sim 690 \mathrm{GeV}$ and quite small $\tan \beta$ values around 1 . Only one point provides an SFOEWPT with a medium charged Higgs boson mass and a $\tan \beta$ value around 5 . All the other points of the second region with larger values of $\tan \beta$ have a charged Higgs boson mass below $200 \mathrm{GeV}$. Compared to our previous analysis [60], parameter points with medium charged Higgs boson masses and large $\tan \beta$ values could not be found any more, so that we have this strict separation of small masses in combination with large $\tan \beta$ values and medium masses in combination with small $\tan \beta$. The maximum strength of the phase transition 
that we found for the C2HDM T1 is $\xi_{c} \approx 1.7$, and for the C2HDM T2 it is $\xi_{c} \approx 1.18$, which is compatible with our findings in [60].

In the C2HDM T2, the flavor constraints $B \rightarrow X_{s} \gamma$ [107-111] require the charged Higgs boson mass to be above $580 \mathrm{GeV}$, which is reflected in figure 1 (right). The figure shows that most parameter points compatible with theoretical and experimental constraints have a rather small $\tan \beta$ of the order of $\mathcal{O}(\tan \beta) \approx 1-4$ and charged Higgs boson masses up to $\sim 1.1 \mathrm{TeV}$. The requirement an SFOEWPT like in the C2HDM T1 sets an upper bound on the charged Higgs boson mass which is $\sim 700 \mathrm{GeV}$. All valid parameter points that have an SFOEWPT gather in the lower left corner of the plot, with small $\tan \beta$ values and as light as possible charged Higgs boson masses. Future updates in the flavor sector that constrain this specific corner of the parameter space might rule out the C2HDM T2 in combination with an SFOEWPT.

Compatibility with the electroweak precision observables enforces the degeneracy of two Higgs boson masses, so either $m_{H^{ \pm}} \approx m_{H_{\downarrow}, H_{\uparrow}, h}$ or a pair of the neutral Higgs bosons are mass degenerate. In order to quantify this effect, we first of all look for that pair of Higgs masses $\left(m_{i}, m_{j}\right)$ that has the minimum mass difference out of all possible Higgs mass pairings, i.e. we define the mass gap of the (almost) degenerate pair $\left(m_{H_{i}}, m_{H_{j}}\right)$ as

$$
\Delta_{m_{H_{i}}, m_{H_{j}}}=\min _{m_{H_{i}} \neq m_{H_{j}}}\left|m_{H_{i}}-m_{H_{j}}\right|, \quad \text { with } H_{i, j} \in\left\{h, H_{\downarrow}, H_{\uparrow}, H^{ \pm}\right\} .
$$

The requirement of an SFOEWPT tightens this mass gap to even smaller values

$$
\begin{gathered}
\text { Type I : } \max _{\text {sample }} \Delta_{m_{H_{i}}, m_{H_{j}}} \approx 61 \mathrm{GeV} \stackrel{\text { EWPT }}{\longrightarrow} \approx 21 \mathrm{GeV}, \\
\text { Type II }: \max _{\text {sample }} \Delta_{m_{H_{i}}, m_{H_{j}}} \approx 62 \mathrm{GeV} \stackrel{\text { EWPT }}{\longrightarrow} \approx 33 \mathrm{GeV} .
\end{gathered}
$$

In both types of the $\mathrm{C} 2 \mathrm{HDM}$ the mass degeneracy that is realized in most of the points is the one of $H_{\downarrow}$ and $H_{\uparrow}$, as shown in figure 2. It shows the non-SM-like Higgs boson mass plane $m_{H_{\uparrow}}-m_{H_{\downarrow}}$ with the color code denoting the values of $\xi_{c}$ above 1 , hence an SFOEWPT. In the other cases the mass degeneracy occurs with the charged Higgs boson, i.e. $m_{H^{ \pm}} \approx m_{H_{\downarrow}}$ or $m_{H^{ \pm}} \approx m_{H_{\uparrow}}$. In the C2HDM T1 in figure 2(a) two mass hierarchies are possible, the heavy mass hierarchy $m_{h}<m_{H_{\downarrow}}<m_{H_{\uparrow}}$ and the fully inverted mass hierarchy $m_{H_{\downarrow}}<m_{H_{\uparrow}}<m_{h}$. As we will see later, in the N2HDM the semi-inverted and the heavy mass hierarchies are possible in $\mathrm{T} 1$ and $\mathrm{T} 2$.

As noted in [45] the SFOEWPT favors a light scalar spectrum, where additional heavy degrees of freedom, that do not obtain an $\mathrm{SU}(2)_{L} \mathrm{VEV}$, can help to strengthen the phase transition. At the same time, the mass degeneracy of $H_{\uparrow}$ and $H_{\downarrow}$ enforces the overall scalar spectrum to be in a medium range of $\mathcal{O}\left(m_{H_{\downarrow}}, m_{H_{\uparrow}}\right) \sim 500 \mathrm{GeV}$. The parameter region with $m_{h}<m_{H_{\downarrow}}<m_{H_{\uparrow}}$ with a heavy $H_{\uparrow}\left(m_{H_{\downarrow}} \ll m_{H_{\uparrow}}\right)$ could also produce an SFOEWPT as remarked in [45], since this region has a light $H_{\downarrow}$ enabling the SFOEWPT and in the meantime the heavy degree of freedom $H_{\uparrow}$ could build up a deep potential barrier between the symmetric and broken minimum. Since this parameter region is restricted by the di-Higgs searches through Higgs decays $H_{\uparrow} \rightarrow H_{\downarrow} H_{\downarrow} / h h$, the parameter space in this region is already sparse due to the collider constraints. Consequently, with the updated 


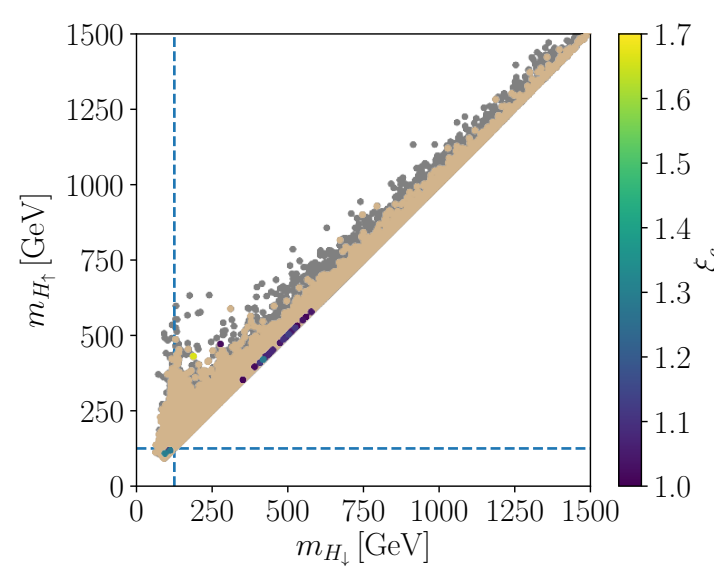

(a)

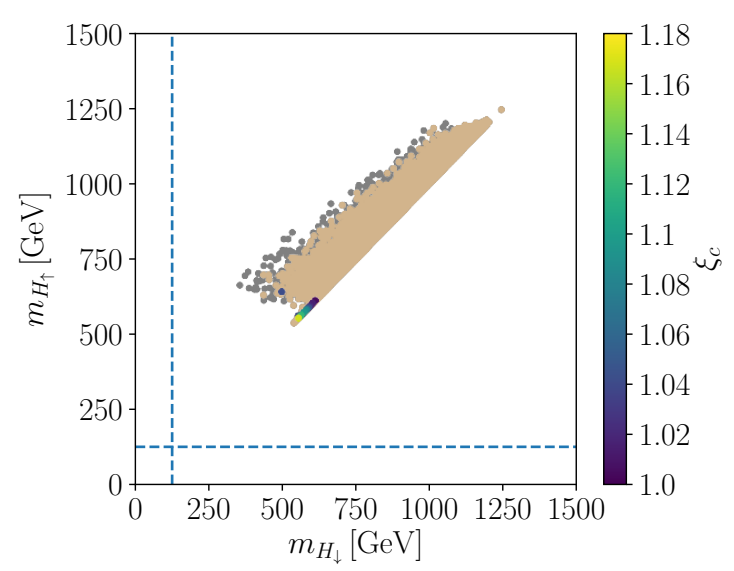

(b)

Figure 2. C2HDM: the mass $m_{H_{\downarrow}}$ versus $m_{H_{\uparrow}}$ for T1 (left) and T2 (right). The grey points are all parameter points passing the experimental and theoretical checks of ScannerS. The color code denotes the strength of the phase transition $\xi_{c}$ for $\xi_{c} \geq 1$. The di-Higgs search constraints are included. The blue dashed lines indicate the SM Higgs mass value of $m_{h} \approx 125 \mathrm{GeV}$.

HiggsSignals 2.3.0 and HiggsBound 5.5.0 versions taking into account the recent diHiggs searches, it is more involved to find parameter points compatible with the collider constraints and an SFOEWPT. As we will see later, this restriction can be circumvented in the N2HDM due to the singlet admixture.

In the C2HDM T2 the overall scalar spectrum in figure 2(b) is heavier compared to T1. This is due to the already required heavy charged Higgs boson mass and the small mass gaps. As the SFOEWPT still favors a light scalar spectrum, the only parameter points providing an SFOEWPT are found in the edge of smallest masses. The overall order of neutral non-SM like Higgs masses providing an SFOEWPT is also $\mathcal{O}\left(m_{h_{i}}\right) \sim 500 \mathrm{GeV}$ as in the C2HDM T1.

To conclude the C2HDM update, we find that the mass spectrum compatible with the recent collider and theoretical constraints, for $\mathrm{T} 1$ is mainly constrained by the recent di-Higgs measurements whereas for T2 the flavor constraints are the most restrictive ones. Future analyses with increasing constraining power in these mass regions might exclude significant regions of the C2HDM parameter space providing an SFOEWPT.

\subsection{N2HDM - phenomenology of the SFOEWPT}

In the following we discuss the implications of an SFOEWPT on the phenomenology of the N2HDM. ${ }^{4}$ The N2HDM has one more degree of freedom compared to the C2HDM due to the additional singlet in the Higgs sector. The larger number of free parameters in this model reduces the influence of the constraints on the parameter space so that heavier Higgs spectra compatible with an SFOEWPT are still possible. In this kind of

\footnotetext{
${ }^{4}$ For a recent study within the NMSSM that is matched to a 2 HDM plus singlet field and can thus be compared to some extent to the N2HDM T2, see [134].
} 


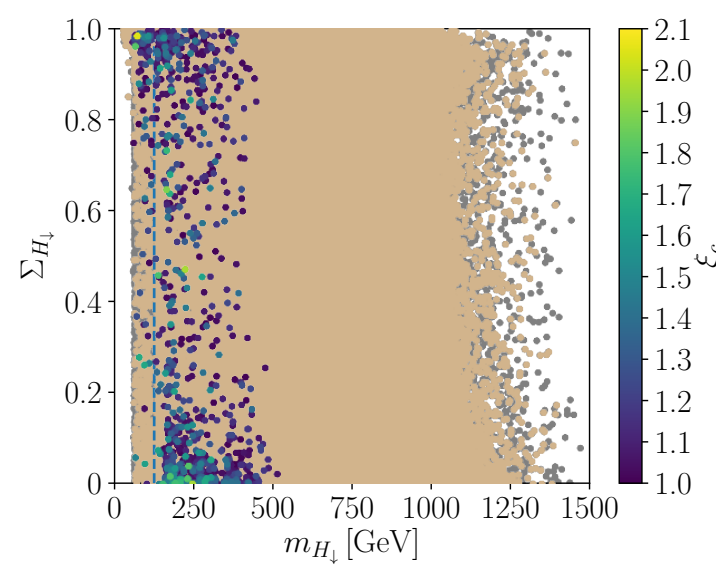

(a)

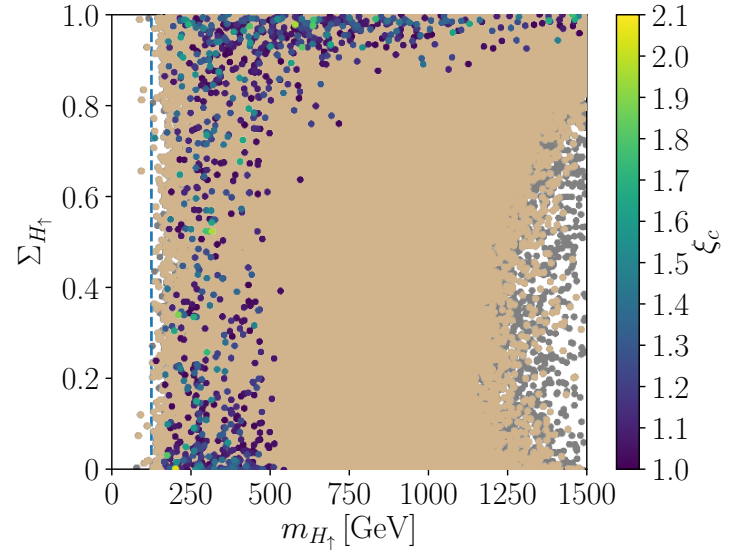

(b)

Figure 3. N2HDM T1: the singlet admixture $\Sigma$ versus the mass for $H_{\downarrow}$ (left) and $H_{\uparrow}$ (right). The grey points are all parameter points passing the experimental and theoretical checks of ScannerS. The brown points additionally fulfill NLO vacuum stability and NLO perturbative unitarity constraints. The color code shows the strength of the phase transition $\xi_{c}$ for $\xi_{c} \geq 1$. The di-Higgs search constraints are included. The blue dashed line indicates the SM Higgs mass value of $m_{h} \approx 125 \mathrm{GeV}$.

scenarios, however, one of the non-SM like Higgs bosons is almost completely singlet-like. The maximum values of $\xi_{c}$ that we find are $\xi_{c}^{\mathrm{N} 2 \mathrm{HDM} \mathrm{T} 1}=2.04$ and $\xi_{c}^{\mathrm{N} 2 \mathrm{HDM}, \mathrm{T} 2}=1.43$ for the N2HDM T1 and T2, respectively. We start with the investigation of the mass spectrum followed by the discussion of the trilinear Higgs self-couplings. Afterwards, we will present several benchmark points providing interesting scenarios with different features.

\subsubsection{Mass spectrum of the N2HDM T1}

For the following discussion of the N2HDM we introduce the singlet admixture $\Sigma_{H_{i}}$ of the respective $\mathrm{CP}$-even Higgs boson $H_{i}$ as

$$
\Sigma_{H_{i}}=R_{i 3}^{2}
$$

It describes the amount of admixture of the singlet field $\rho_{S}$ to the corresponding mass eigenstate $H_{i}$. In figure 3 left (right) we plot the singlet admixture $\Sigma_{H_{\downarrow}}\left(\Sigma_{H_{\uparrow}}\right)$ of $H_{\downarrow}$ $\left(H_{\uparrow}\right)$ versus its mass. The grey points denote parameters points compatible with the theoretical and experimental constraints, the brown points additionally provide an NLO stable vacuum and NLO perturbative unitarity. The color code indicates the strength of the phase transition for $\xi_{c} \geq 1$. The masses that provide an SFOEWPT are

$$
m_{H_{\downarrow}} \in[53,513] \mathrm{GeV}, \quad m_{H_{\uparrow}} \in[136,1479] \mathrm{GeV} .
$$

As these mass windows show, in the N2HDM T1 the heavy mass hierarchy $\left(m_{h}<m_{H_{\downarrow}}<\right.$ $\left.m_{H_{\uparrow}}\right)$ and the semi-inverted mass hierarchy $\left(m_{H_{\downarrow}}<m_{h}<m_{H_{\uparrow}}\right)$ are possible, whereas the inverted hierarchy $\left(m_{H_{\downarrow}}<m_{H_{\uparrow}}<m_{h}\right)$ is not realised. We will provide benchmark scenarios 


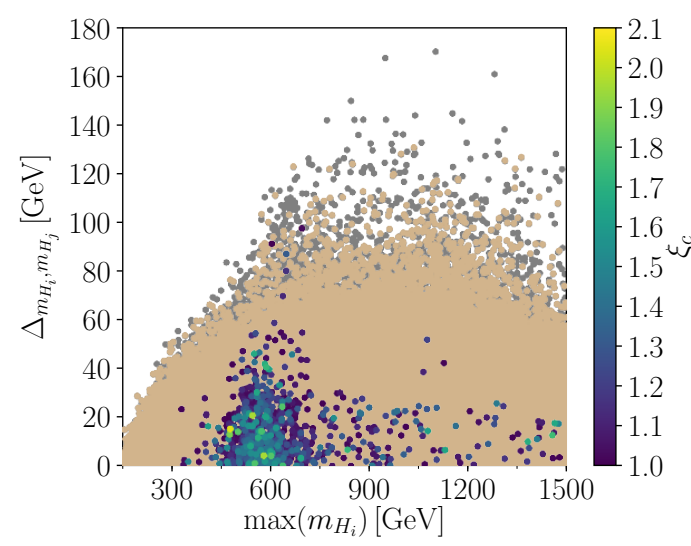

(a)

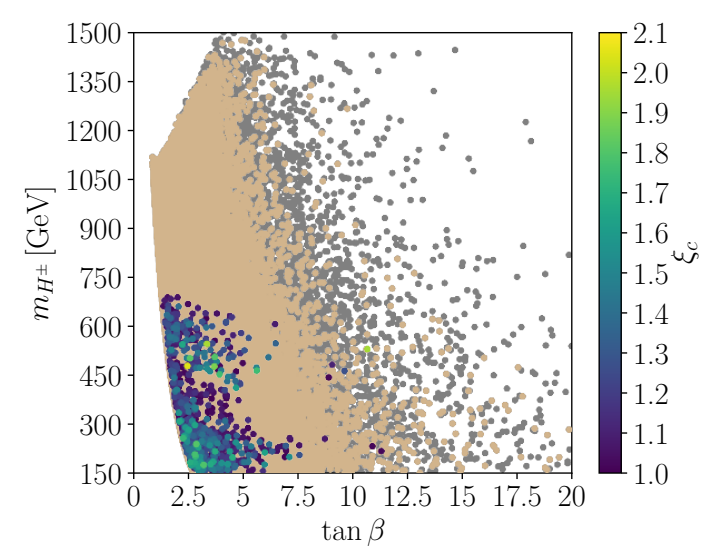

(b)

Figure 4. N2HDM T1: Left: the minimum mass gap $\Delta_{m_{H_{i}}, m_{H_{j}}}$ versus the maximum of the scalar masses. Right: charged Higgs boson mass $m_{H^{ \pm}}$versus $\tan \beta$. The color code is the same as in figure 3. The di-Higgs search constraints are included.

for all possible cases. Like in the C2HDM, an SFOEWPT favors light Higgs mass spectra below $\sim 500 \mathrm{GeV}$ except for spectra with singlet-like $H_{\uparrow}$. If the heaviest CP-even non-SM like Higgs boson $H_{\uparrow}$ has a singlet admixture above $\Sigma_{H_{\uparrow}} \gtrsim 80 \%$, the SFOEWPT opens the window for larger masses $m_{H_{\uparrow}}$. In case of singlet admixtures below about $80 \%, \Sigma_{H_{\uparrow}} \lesssim 80 \%$, on the other hand the same preference for intermediate Higgs mass spectra as in the $\mathrm{C} 2 \mathrm{HDM}$ can be observed for $\xi_{c} \geq 1$. The possibility of large neutral Higgs boson masses allows for heavy Higgs decays into pairs of lighter Higgs bosons, $H_{\uparrow} \rightarrow H_{i} H_{j}$. Since almost all of these heavy states are singlet-like, their couplings to SM particles are suppressed and the Higgs-to-Higgs decay channel becomes an important discovery channel. We will provide benchmark scenarios where these channels may become accessible at the LHC.

As already mentioned, compatibility with the EW precision data checked through the $S, T$ and $U$ parameters requires a small mass gap between the charged Higgs boson $H^{ \pm}$and one of the neutral Higgs bosons or between two neutral Higgs bosons, and the SFOEWPT enforces even more the mass degeneracy between at least one non-SM like Higgs boson pair. In figure 4(a) we show the minimum mass gap out of all possible neutral Higgs pairings, $\Delta_{m_{H_{i}}, m_{H_{j}}}$, defined analogously to eq. (5.1),

$$
\Delta_{m_{H_{i}}, m_{H_{j}}}=\min _{i \neq j}\left|m_{H_{i}}-m_{H_{j}}\right| \quad \text { with } H_{i, j} \in\left\{h, H_{\downarrow}, H_{\uparrow}, H^{ \pm}, A\right\},
$$

versus the maximum mass in the spectrum, $\max \left(m_{H_{i}}\right)$. The color code is the same as in figure 3. The experimental and theoretical constraints allow for mass gaps even above $150 \mathrm{GeV}$ and the NLO stable vacuum and NLO perturbative unitarity are compatible with mass gaps up to about $130 \mathrm{GeV}$, while the SFOEWPT reduces this upper bound down to $\mathcal{O}(50 \mathrm{GeV})$ with a few exceptions of up to $100 \mathrm{GeV}$. Scenarios with degenerate neutral Higgs boson masses are rather rare so that in general the charged Higgs boson mass lies in the same region as the neutral Higgs boson masses. Consequently, the mass spectrum of the charged Higgs boson is also reduced in case of an SFOEWPT. This is reflected in 


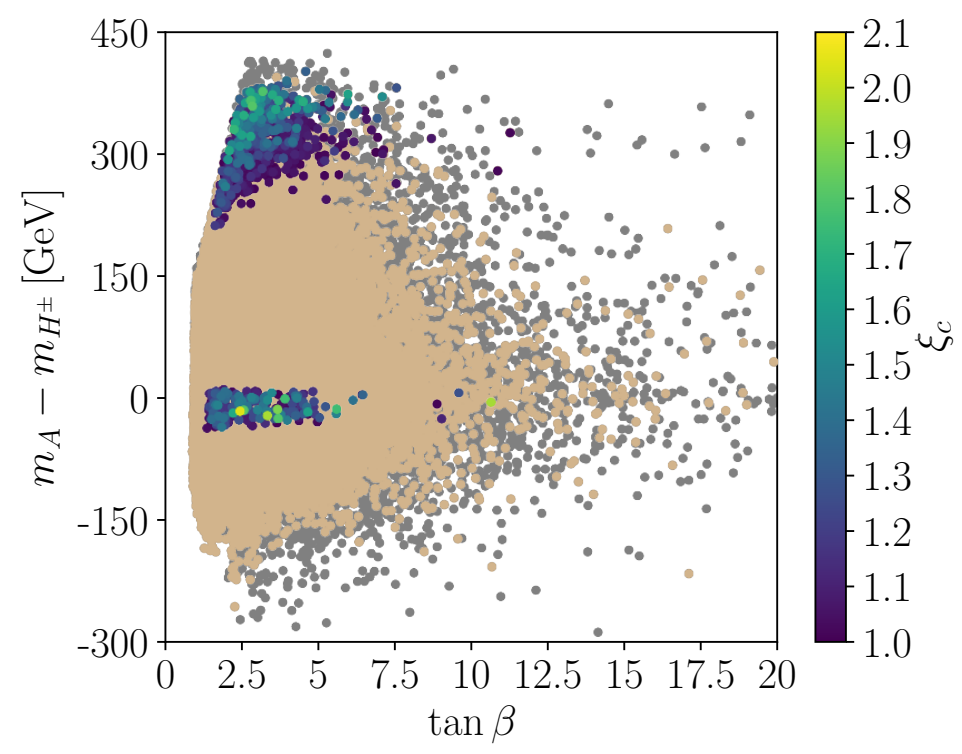

Figure 5. N2HDM T1: the mass difference $m_{A}-m_{H^{ \pm}}$versus $\tan \beta$. The color code is the same as in figure 3. The di-Higgs search constraints are included.

figure 4(b) where the charged mass $m_{H^{ \pm}}$is depicted versus $\tan \beta$, with the same color coding as in figure 4(a).

The last phenomenological effect on the N2HDM mass spectrum induced by the SFOEWPT we want to discuss, is shown in figure 5 . It displays the mass difference $m_{A}-m_{H^{ \pm}}$versus $\tan \beta$. As can be inferred from the figure, the SFOEWPT cuts out two distinct regions in the viable parameter space, $\mathcal{M}_{\text {deg }}$ and $\mathcal{M}_{\text {sep }}$, with

$$
\mathcal{M}_{\mathrm{deg}}: m_{A} \approx m_{H^{ \pm}} \quad \text { and } \quad \mathcal{M}_{\mathrm{sep}}: m_{A} \geq m_{H^{ \pm}}+220 \mathrm{GeV}
$$

This plots shows that an SFOEWPT does not only allow for scenarios with $m_{A} \approx m_{H^{ \pm}}$, but also mass spectra with large gaps between $m_{A}$ and $m_{H^{ \pm}}$are allowed. This possibility should also be taken into account when the EWPT in investigated. For parameter points in $\mathcal{M}_{\mathrm{deg}}$, stronger EWPTs with $\xi_{c}$ up to $\xi_{c} \lesssim 2.1$ can be observed compared to the points in $\mathcal{M}_{\text {sep }}$ with $\xi_{c} \lesssim 1.93$. To discuss the slight tendency of a stronger EWPT in $\mathcal{M}_{\text {sep }}$ for increasing mass gaps $m_{A}-m_{H^{ \pm}}$, we first note the observations made in refs. [60, 78]: the strength of the phase transition $\xi_{c}$ increases with the size of the couplings of the light bosonic particles to the SM-like Higgs boson and decreases with the Higgs boson mass. Additionally, particles that contribute to the EWPT necessarily have a non-vanishing electroweak VEV. All nonSM-like neutral Higgs bosons $H_{\downarrow}, H_{\uparrow}$ receive an electroweak VEV through mixing and therefore, for an SFOEWPT, their masses $m_{H_{\downarrow}}, m_{H_{\uparrow}}$ have to be either light or their VEV has to be small. We note here again explicitly that we take the singlet VEV $v_{S}$ into account for the minimisation of the effective potential, but we do not include $v_{S}$ in the calculation of the electroweak VEV in eq. (4.2). So the EWPT is not directly affected by the singlet VEV, just indirectly through the minimisation. Thus particles that do not obtain an electroweak $\operatorname{VEV}\left(A, H^{ \pm}\right)$and singlet-like $\mathrm{CP}$-even neutral Higgs bosons $\left(\Sigma_{i} \approx 1\right)$ are still allowed to 


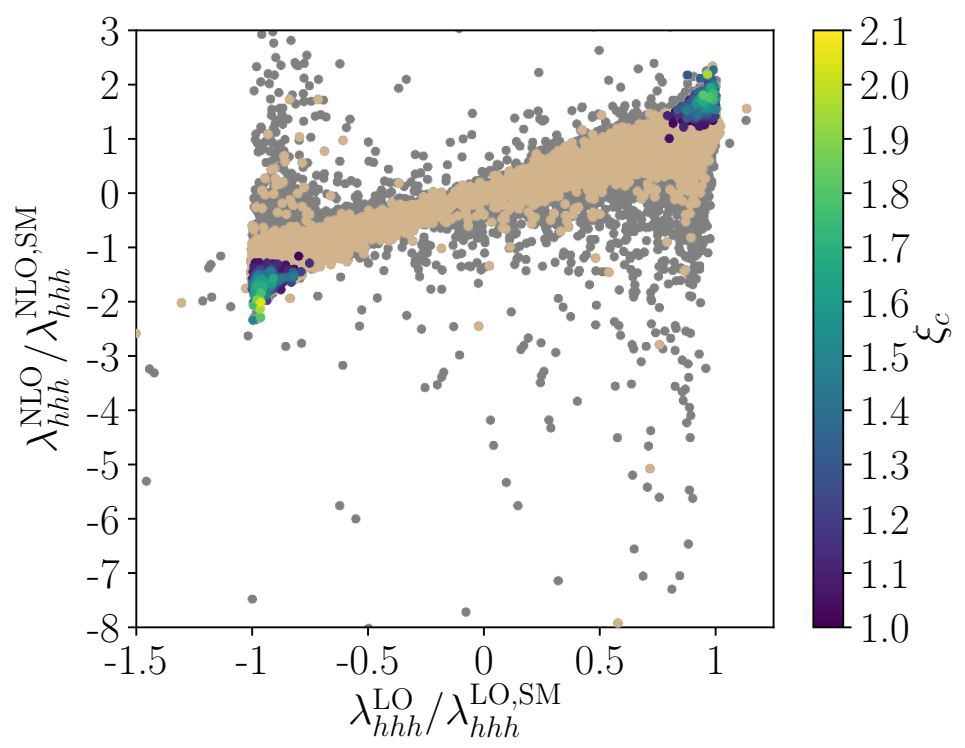

Figure 6. N2HDM T1: the leading-order trilinear self-coupling between three SM-like Higgs bosons normalised to the SM reference value versus the NLO trilinear self-coupling between three SM-like Higgs bosons normalised to the SM reference value. The color code is the same as in figure 3. The di-Higgs search constraints are included.

be heavy without decreasing the strength of the EWPT. The heavy degrees of freedom even help to strengthen the EWPT by enabling a deeper potential barrier between the broken and symmetric phase [47]. For increasing mass gaps $m_{A}-m_{H^{ \pm}}$and therefore increasing $m_{A}$ masses, we have an additional heavy degree of freedom in the spectrum, whereas the bosonic degrees of freedom that obtain an electroweak VEV remain light. Consequently, the strength of the EWPT will increase for the parameter points in $\mathcal{M}_{\text {sep }}$ with increasing mass gap. Since there are additional interplays between $\xi_{c}$ and the mass spectrum or other effects, the effect on the size of $\xi_{c}$ is not significantly enhanced, however.

\subsubsection{N2HDM T1 — trilinear Higgs self-couplings}

The one-loop-corrected trilinear Higgs self-couplings are obtained from the one-loop effective potential by performing the third derivative with respect to the Higgs fields. The problem of infrared divergences related to the Goldstone bosons in the Landau gauge is treated analogously to the extraction of the masses from the second derivative of the potential, cf. ref. [96] for details. In figure 6 the next-to-leading order (NLO) trilinear self-coupling between three SM-like Higgs bosons normalised to the SM reference is plotted versus the leading-order (LO) coupling. The color code is the same as in the previous plots. For the SM reference we take the value of [135] which takes into account the dominant NLO top-quark contribution. The NLO corrections can both suppress and enhance the LO values quite significantly. The experimental and theoretical constraints allow for a largely enhanced NLO Higgs self-coupling compared to the SM NLO value, between a factor of -7.9 and 2.4. By requiring an SFOEWPT this upper bound is reduced down to a 
factor \pm 2.4 . At the same time the SFOEWPT disfavors trilinear self-couplings below the SM value, hence

$$
\lambda_{h h h}^{\mathrm{NLO}} / \lambda_{h h h}^{\mathrm{NLO}, \mathrm{SM}} \in[-2.4,-1.2] \cup[1.0,2.4], \quad \text { for } \xi_{c} \geq 1 .
$$

As observed in [60] the SFOEWPT favors large trilinear Higgs self-couplings which is also observed here, since the strongest EWPTs are located at the maximum values of the trilinear Higgs self-couplings. On the other hand, the upper bound of the trilinear Higgs self-coupling is significantly reduced by the SFOEWPT which can be explained by the interplay between the quartic coupling and the masses of the Higgs bosons participating in the EWPT. Note, that besides the dominant top-loop contributions to the NLO coupling, also the Higgs-loop contributions present in the C2HDM and N2HDM can be large. The masses of the heavy N2HDM Higgs bosons $\Phi$ can be cast in the following schematic form (see [135] for the 2HDM)

$$
m_{\Phi}^{2}=M^{2}+f_{v}\left(\lambda_{i}\right) v^{2}+f_{m}\left(\lambda_{i}\right) v v_{s}+f_{s}\left(\lambda_{i}\right) v_{s}^{2}+\mathcal{O}\left(v^{4} / M^{2}, v_{s}^{4} / M^{2}\right)
$$

with $M^{2}$ denoting the mass scale independent of the VEVs and $f_{v, m, s}\left(\lambda_{i}\right)$ a linear combination of the quartic couplings of the Higgs potential. For an SFOEWPT large couplings $\lambda_{i}$ are required. On the other hand, the masses should not become too heavy, which we observed in the previous discussion of the general mass spectrum, thus limiting the maximum values for the quartic coupling due to eq. (5.9). This explains why we observe the strongest EWPT for the largest trilinear coupling, but the maximum enhancement of the trilinear Higgs self-coupling for a strong EWPT remains below the value compatible with the applied constraints.

Since the N2HDM, in contrast to the C2HDM, has a singlet VEV which does not contribute to the EWPT, heavy degrees of freedom are compatible with an SFOEWPT if they are singlet-like. In figure 7(a) we display the NLO trilinear Higgs self-coupling between three heavy Higgs bosons $H_{\uparrow}$ normalised to the NLO trilinear Higgs self-coupling of the SM versus one minus the singlet admixture $\Sigma_{H_{\uparrow}}$ of $H_{\uparrow}$ (which corresponds to the doublet admixture of $H_{\uparrow}$ ). The meaning for the grey and brown points is the same as in the previous plots. The colored points now, however, denote the mass value $m_{H_{\uparrow}}$ for those parameter points that are compatible with an SFOEWPT. We observe that all singlet admixtures from $0 \%$ up to $100 \%$ are possible for intermediate mass ranges, but only for the singlet-like heavy states we observe large masses $m_{H_{\uparrow}}$ of up to $1490 \mathrm{GeV}$. For these heavy masses the trilinear self-coupling $\lambda_{H_{\uparrow} H_{\uparrow} H_{\uparrow}}^{\mathrm{NLO}}$ is enhanced compared to the parameter points with intermediate masses. Simultaneously these large masses enhance the trilinear coupling $\lambda_{h h H_{\uparrow}}$ opening a window for heavy Higgs decay chains like $H_{\uparrow} \rightarrow h h \rightarrow X X$. The enhancement can be observed in figure $7(\mathrm{~b})$ where the NLO trilinear self-coupling $\lambda_{h h H_{\uparrow}}$ normalised to the NLO trilinear Higgs self-coupling of the SM is plotted against one minus the singlet admixture $\Sigma_{H_{\uparrow}}$, with the same color code as in figure 7 (a). Like for the self-coupling $\lambda_{H_{\uparrow} H_{\uparrow} H_{\uparrow}}$ the largest enhancements of $\lambda_{h h H_{\uparrow}}$ compatible with an SFOEWPT are observed for large singlet admixtures. These heavy Higgs decays allow to distinguish between the C2HDM and the N2HDM as we will discuss later. 


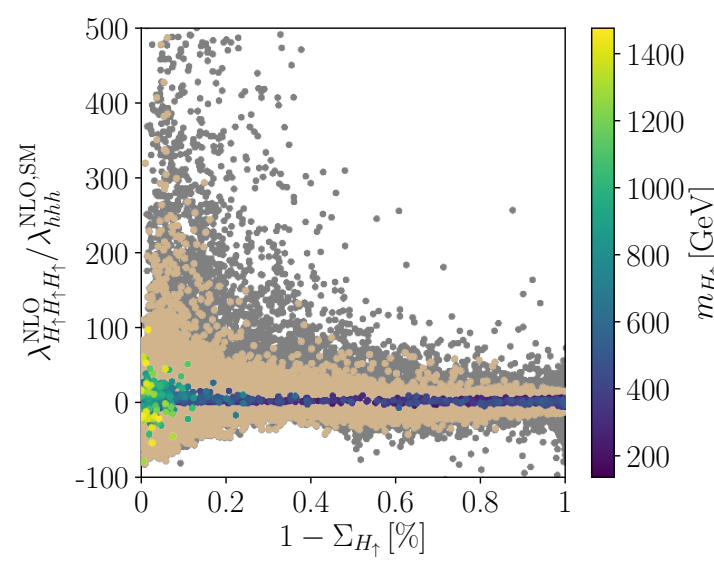

(a)

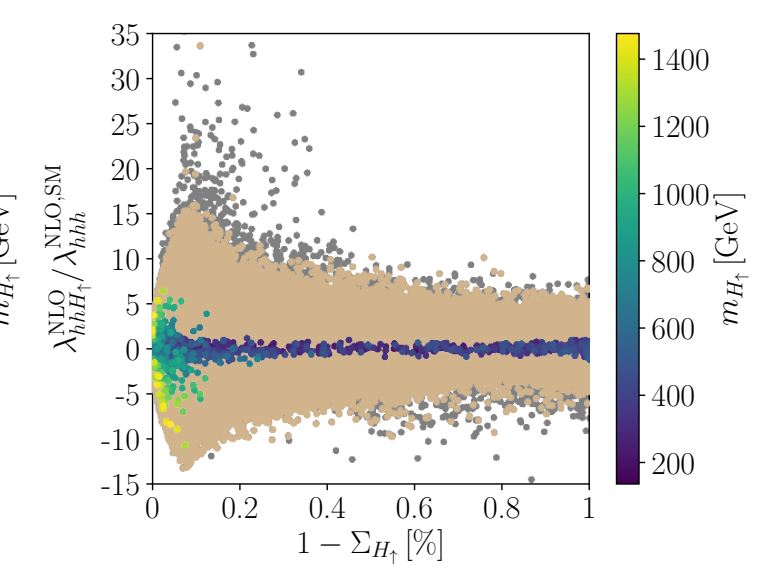

(b)

Figure 7. N2HDM T1: Left: the NLO trilinear self-coupling between three heavy neutral Higgs bosons $H_{\uparrow}$ normalised to the SM reference value versus the doublet admixture of the heavy neutral Higgs boson $\Sigma_{H_{\uparrow}}$. Right: the NLO trilinear self-coupling between two SM-like Higgs bosons and one heavy neutral Higgs boson $H_{\uparrow}$ normalised to the NLO SM reference value. The color scale show the mass value $m_{H_{\uparrow}}$ for those points that are compatible with an SFOEWPT.

\begin{tabular}{|cccccccccc|}
\hline & $m_{H_{\downarrow}}$ & $m_{H_{\uparrow}}$ & $m_{H^{ \pm}}$ & $m_{A}$ & $\tan \beta$ & $\alpha_{1}$ & $\alpha_{2}$ & $\alpha_{3}$ & $v_{S}$ \\
\hline BPSep & 101.22 & 270.97 & 230.89 & 558.03 & 2.462 & -0.721 & 1.062 & 0.213 & 705.78 \\
BPDeg & 67.00 & 178.76 & 348.95 & 350.68 & 2.826 & -0.388 & -0.243 & -0.052 & 1723 \\
\hline & $\lambda_{1}$ & $\lambda_{2}$ & $\lambda_{3}$ & $\lambda_{4}$ & $\lambda_{5}$ & $\lambda_{6}$ & $\lambda_{7}$ & $\lambda_{8}$ & $m_{12}^{2}$ \\
\hline BPSep & 3.3400 & 0.4038 & 0.5121 & 3.7907 & -4.7239 & 0.0494 & -0.3549 & 0.0786 & 8722.03 \\
BPDeg & 0.8643 & 0.2589 & 4.1685 & -1.9557 & -1.9957 & 0.0102 & 0.0441 & -0.0042 & 625.03 \\
\hline
\end{tabular}

Table 7. N2HDM T1 benchmark points BPSep and BPDeg with semi-inverted mass hierarchy. The masses and the singlet VEV $v_{s}$ are given in $[\mathrm{GeV}]$.

\subsubsection{N2HDM T1 - benchmark points}

In the following we will present benchmark points that provide an SFOEWPT and have interesting phenomenological features. In total we generated 271743 parameter points with ScannerS, fulfilling theoretical and experimental constraints. After applying the NLO constraints, NLO vacuum stability and NLO unitarity, and demanding an SFOEWPT 920 parameter points are left in the sample. 836 of these points feature the heavy mass hierarchy while 84 have the semi-inverted.

Semi-inverted mass hierarchy. In table 7 two benchmark points are listed with a semi-inverted mass hierarchy where

$$
m_{H_{\downarrow}} \lesssim m_{h} \lesssim m_{H_{\uparrow}} .
$$

The first parameter point BPSep is in the region of the parameter space where the pseudoscalar mass and the charged mass have a large mass gap, denoted by $\mathcal{M}_{\text {sep }}$ in the 
previous discussion. The neutral $\mathrm{CP}$-even and the charged Higgs boson masses are all light and below about $271 \mathrm{GeV}$ whereas the pseudoscalar mass is significantly heavier with a mass of $558 \mathrm{GeV}$. BPsep provides a rather strong SFOEWPT with $\xi_{c}=1.31$ $\left(T_{c}=133.21 \mathrm{GeV}, v_{c}=174.59 \mathrm{GeV}\right)$. This is in agreement with the already discussed observation that a light mass spectrum in combination with a heavy scalar degree of freedom strengthens the phase transition. The singlet admixtures of the neutral non-SM-like CP-even Higgs bosons of BPSep are

$$
\Sigma_{H_{\downarrow}}=76.3 \%, \quad \Sigma_{H_{\uparrow}}=22.7 \%
$$

The mass hierarchy allows for the Higgs-to-Higgs decays $H_{\uparrow} \rightarrow H_{\downarrow} h, H_{\uparrow} \rightarrow H_{\downarrow} H_{\downarrow}$ and $H_{\uparrow} \rightarrow h h$ with branching ratios of

$$
\mathrm{BR}\left(H_{\uparrow} \rightarrow H_{\downarrow} h\right)=74.6 \%, \mathrm{BR}\left(H_{\uparrow} \rightarrow H_{\downarrow} H_{\downarrow}\right)=13.2 \%, \mathrm{BR}\left(H_{\uparrow} \rightarrow h h\right)=2.5 \%
$$

With $H_{\uparrow}$ being doublet-like and rather light also the production cross section is reasonably large so that for a c.m. energy of $\sqrt{s}=13 \mathrm{TeV}$ we have a signal rate of

$$
\sigma\left(p p \rightarrow H_{\uparrow} \rightarrow\left(H_{\downarrow} \rightarrow \bar{b} b\right)(h \rightarrow \bar{b} b)\right)=740.3 \mathrm{fb} .
$$

This is phenomenologically very interesting, as we have two different Higgs boson masses in the final state and a rather large cross section. To put this into context, we remind the reader that the production cross section for a pair of SM-like Higgs bosons including NLO QCD corrections taking into account the full top-quark mass dependence is $32.91 \mathrm{fb}$ at $\sqrt{s}=14 \mathrm{TeV}[136-138]$.

The second parameter point BPDeg belongs to the phase space region $\mathcal{M}_{\mathrm{deg}}$ where $m_{A} \approx m_{H^{ \pm}}$and also provides a rather high $\xi_{c}=1.38\left(T_{c}=135.92 \mathrm{GeV}, v_{c}=188.17 \mathrm{GeV}\right)$. While the non-SM-like CP-even Higgs bosons are somewhat lighter than in BPSep, the pseudoscalar and charged Higgs boson masses are around $350 \mathrm{GeV}$, so that the decay of $A$ into a gauge plus Higgs boson pair is kinematically possible. With the singlet admixtures of $H_{\uparrow}$ and $H_{\downarrow}$ given by

$$
\Sigma_{H_{\downarrow}}=5.8 \%, \quad \Sigma_{H_{\uparrow}}=94.0 \%,
$$

the dominant decay is into the doublet-like Higgs, hence $A \rightarrow Z H_{\downarrow}$. The parameter point has been chosen as it provides the largest signal rate for $A \rightarrow Z H_{\downarrow}$ among our parameter sample, with

$$
\sigma\left(p p \rightarrow A \rightarrow Z H_{\downarrow}\right)=6.91 \mathrm{pb}
$$

at $\sqrt{s}=13 \mathrm{TeV}$. This signature would be a clear sign of beyond-the-SM physics and should be accessible at the LHC in view of the large cross section. We add that the dominant branching ratios of $H_{\downarrow}$ are given by

$$
\mathrm{BR}\left(H_{\downarrow} \rightarrow \bar{b} b\right)=84.6 \%, \quad \mathrm{BR}\left(H_{\downarrow} \rightarrow \tau \tau\right)=7 \% .
$$




\begin{tabular}{|cccccccccc|}
\hline & $m_{H_{\downarrow}}$ & $m_{H_{\uparrow}}$ & $m_{H^{ \pm}}$ & $m_{A}$ & $\tan \beta$ & $\alpha_{1}$ & $\alpha_{2}$ & $\alpha_{3}$ & $v_{S}$ \\
\hline BPii1 & 285.26 & 1461.94 & 543.24 & 525.72 & 2.226 & 1.189 & 0.081 & 0.072 & 757.08 \\
BPii2 & 221.71 & 269.93 & 217.75 & 570.913 & 6.522 & 1.319 & -0.227 & -0.387 & 945.55 \\
\hline & $\lambda_{1}$ & $\lambda_{2}$ & $\lambda_{3}$ & $\lambda_{4}$ & $\lambda_{5}$ & $\lambda_{6}$ & $\lambda_{7}$ & $\lambda_{8}$ & $m_{12}^{2}$ \\
\hline BPii1 & 0.1406 & 0.6336 & 6.9388 & -3.7178 & -3.1000 & 3.6855 & 0.9530 & -1.2625 & 33065.00 \\
BPii2 & 2.5074 & 0.3431 & -0.3006 & 4.5889 & -4.5997 & 0.0747 & -0.1379 & 0.0585 & 7054.28 \\
\hline
\end{tabular}

Table 8. N2HDM T1 benchmark points BPii1 and BPii2 with heavy mass hierarchy. The masses and the singlet VEV $v_{s}$ are given in $[\mathrm{GeV}]$.

Heavy mass hierarchy. In table 8 , we provide two benchmark points for the heavy mass hierarchy. The parameter point BPiil features a quite heavy $H_{\uparrow}$, while the other Higgs-boson masses are in the intermediate mass range so that the mass gap between the neutral non-SM like Higgs bosons $H_{\uparrow}$ and $H_{\downarrow}$ is very large. In contrast, BPii2 has an overall light Higgs spectrum apart from the pseudoscalar Higgs boson with a mass of $571 \mathrm{GeV}$. For both benchmark points the singlet admixture of the heavy CP-even Higgs boson is quite high so that $H_{\uparrow}$ is singlet-like with

$$
\Sigma_{H_{\uparrow}}^{\mathrm{BPii} 1}=98.8 \% \quad \text { and } \quad \Sigma_{H_{\uparrow}}^{\mathrm{BPii} 2}=81.1 \% .
$$

The enhanced mass of $H_{\uparrow}$ with $m_{H_{\uparrow}}=1.46 \mathrm{TeV}$ of BPii1 is only possible for an almost completely singlet-like state, so that this heavy degree of freedom does not contribute to the EWPT. This allows us to have a strong EWPT with $\xi_{c}=1.66\left(T_{c}=130.1 \mathrm{GeV}, v_{c}=\right.$ $216.3 \mathrm{GeV}$ ). The benchmark point BPii2 with an intermediate Higgs mass spectrum only has a $\xi_{c}=1.04\left(T_{c}=136.54 \mathrm{GeV}, v_{c}=141.96 \mathrm{GeV}\right)$. Among the parameter points with this mass hierarchy, BPii2 has the largest signal rate for the production of a pair of SMlike Higgs bosons through $H_{\uparrow} \rightarrow h h$. It has a larger branching ratio for this final state than BPii1, with $\mathrm{BR}\left(H_{\uparrow} \rightarrow h h\right)_{\mathrm{BPii} 2}=31.3 \%$ compared to $\mathrm{BR}\left(H_{\uparrow} \rightarrow h h\right)_{\mathrm{BPii} 1}=10.9 \%$ in BPii1, and the gluon fusion cross section is larger because of the lighter mass $m_{H_{\uparrow}}$ compared to BPii1,

$$
\sigma\left(g g \rightarrow H_{\uparrow}\right)_{\mathrm{BPii} 1}=0.13 \mathrm{fb} \quad \text { and } \quad \sigma\left(g g \rightarrow H_{\uparrow}\right)_{\mathrm{BPii} 2}=1.01 \mathrm{pb}
$$

for a c.m. energy of $\sqrt{s}=13 \mathrm{TeV}$. We then have for BPii2 the following signal rates for SM-like di-Higgs production in the $4 b,(\bar{b} b)(\tau \tau),(\bar{b} b)(W W), 4 W$ and $(\bar{b} b)(\gamma \gamma)$ final states,

$$
\begin{aligned}
\sigma\left(p p \rightarrow H_{\uparrow} \rightarrow(h \rightarrow \bar{b} b)(h \rightarrow \bar{b} b)\right) & =109.3 \mathrm{fb} \\
\sigma\left(p p \rightarrow H_{\uparrow} \rightarrow(h \rightarrow \bar{b} b)(h \rightarrow \bar{\tau} \tau)\right) & =11.8 \mathrm{fb} \\
\sigma\left(p p \rightarrow H_{\uparrow} \rightarrow(h \rightarrow \bar{b} b)(h \rightarrow W W)\right) & =39.9 \mathrm{fb} \\
\sigma\left(p p \rightarrow H_{\uparrow} \rightarrow(h \rightarrow W W)(h \rightarrow W W)\right) & =14.6 \mathrm{fb} \\
\sigma\left(p p \rightarrow H_{\uparrow} \rightarrow(h \rightarrow \bar{b} b)(h \rightarrow \gamma \gamma)\right) & =0.4 \mathrm{fb} .
\end{aligned}
$$

In BPii1, due to the smaller $H_{\uparrow}$ production cross section we have for the $4 b$ final state the much smaller rate

$$
\sigma\left(p p \rightarrow H_{\uparrow} \rightarrow(h \rightarrow \bar{b} b)(h \rightarrow \bar{b} b)\right)=0.005 \mathrm{fb} .
$$




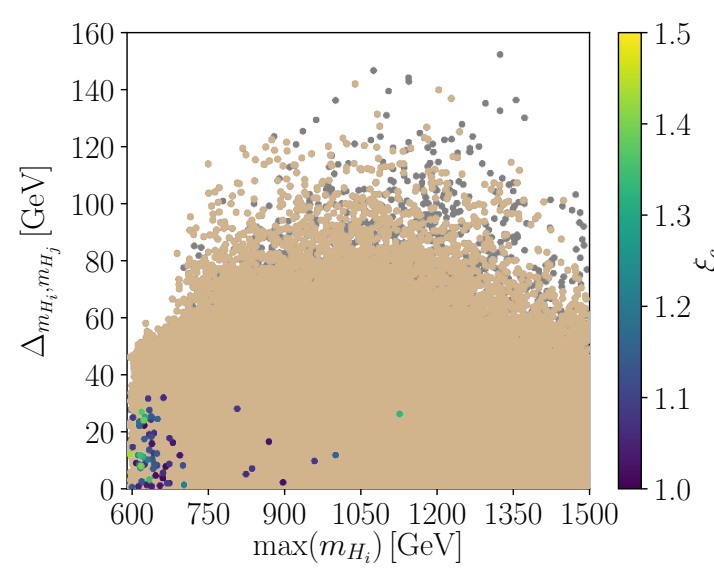

(a)

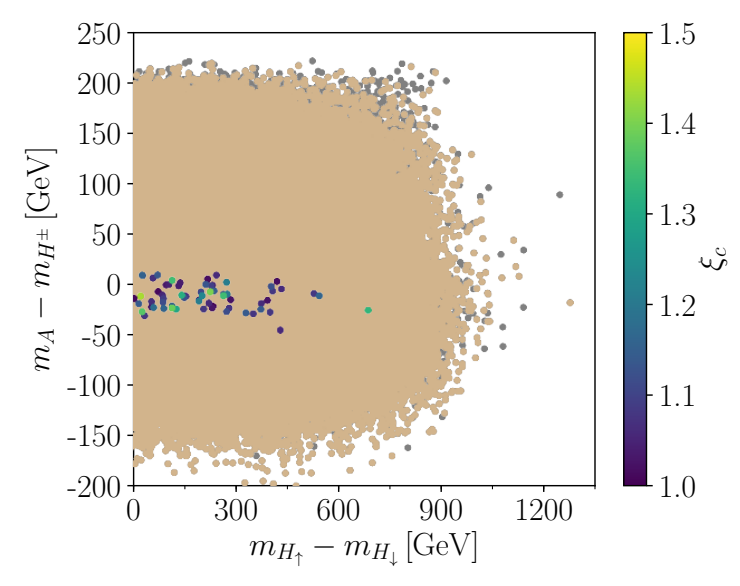

(b)

Figure 8. N2HDM T2: Left: the minimum mass gap $\Delta_{m_{H_{i}}, m_{H_{j}}}$ versus the maximum of the scalar masses. Right: the mass gap between the CP-odd and charged Higgs bosons, $A$ and $H^{ \pm}$, versus the mass gap between the CP-even neutral Higgs bosons $H_{\uparrow}$ and $H_{\downarrow}$. The color code is the same as in figure 3. The color bar indicates the strength of the phase transition for $\xi_{c} \geq 1$. The di-Higgs search constraints are included.

The benchmark scenario BPii1 features enhanced trilinear Higgs self-couplings $\lambda_{h h h}^{\mathrm{NLO}, \mathrm{N} 2 \mathrm{HDM}} / \lambda_{h h h}^{\mathrm{NLO}, \mathrm{SM}}=1.93$ between three SM-like Higgs bosons, and the largest absolute value for the coupling between $H_{\uparrow}$ and two SM-like Higgs bosons $h$ of the sample, with $\lambda_{h h H_{\uparrow}}^{\mathrm{NLO}, \mathrm{N} 2 \mathrm{HDM}} / \lambda_{h h h}^{\mathrm{NLO}, \mathrm{SM}}=-3.97$. Despite the significantly enhanced trilinear Higgs self-couplings the expected signals in the di-Higgs rates are small due to the small production cross section of the heavy Higgs state. On the other hand, BPii2 with its trilinear Higgs self-couplings of

$$
\lambda_{h h h}^{\mathrm{NLO}, \mathrm{N} 2 \mathrm{HDM}} / \lambda_{h h h}^{\mathrm{NLO}, \mathrm{SM}}=-1.47, \quad \lambda_{h h H_{\uparrow}}^{\mathrm{NLO}, \mathrm{N} 2 \mathrm{HDM}} / \lambda_{h h h}^{\mathrm{NLO}, \mathrm{SM}}=0.67,
$$

allows for the largest expected di-Higgs signals in $p p \rightarrow H_{\uparrow} \rightarrow(h \rightarrow X)(h \rightarrow Y)$. To conclude, singlet-like ${ }^{5}$ heavy Higgs bosons are interesting in the sense that they can strengthen the EWPT by providing a heavy scalar degree of freedom whereby the remaining neutral Higgs bosons can have light or intermediate mass values. Those heavy degrees of freedom can have significantly enhanced couplings to the SM-like Higgs boson. Simultaneously the production cross-section of the heavy state is reduced, however, so that the expected diHiggs signals are suppressed. After all there exist also points having a compromise with an intermediate mass spectrum and decent up to large expected signals like BPii2.

\subsubsection{Mass spectrum of the N2HDM T2}

We start our discussion of the N2HDM T2 mass spectrum with the minimum mass gap $\Delta_{m_{H_{i}}, m_{H_{j}}}$ among all possible pairings of the N2HDM Higgs bosons. Figure 8(a) shows

\footnotetext{
${ }^{5}$ Singlet-like Higgs states do not directly contribute to the EWPT since the singlet VEV $v_{S}$ is not included in the determination of $\omega_{c}$.
} 


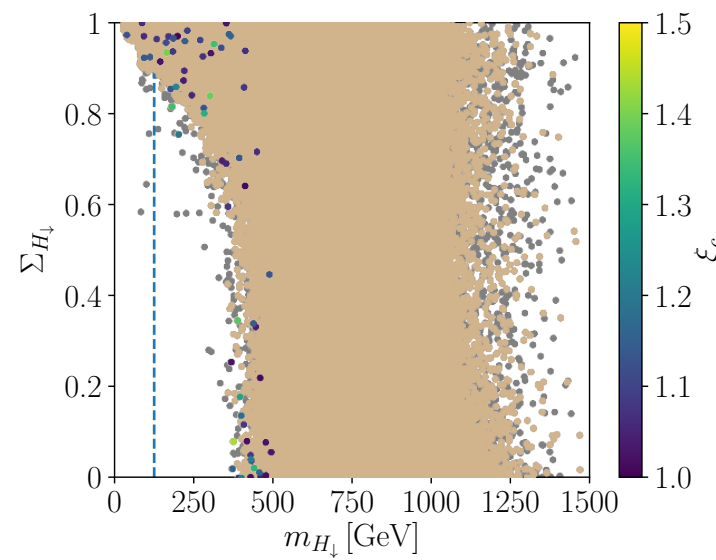

(a)

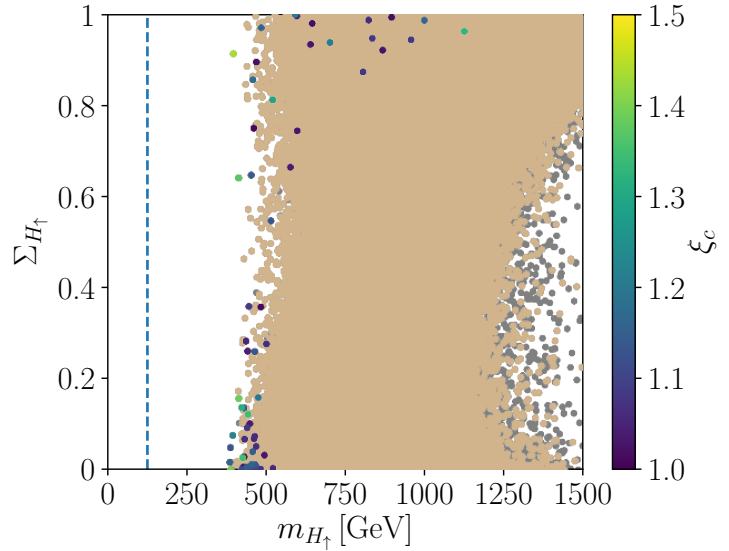

(b)

Figure 9. N2HDM T2: Left: singlet admixture $\Sigma_{H_{\downarrow}}$ versus $m_{H_{\downarrow}}$; Right: $\Sigma_{H_{\uparrow}}$ versus $m_{H_{\uparrow}}$. The color code is the same as in figure 3. The color bar indicates the strength of the phase transition for $\xi_{c} \geq 1$. The di-Higgs search constraints are included. The blue dashed line indicates the SM Higgs mass value of $m_{h} \approx 125 \mathrm{GeV}$.

$\Delta_{m_{H_{i}}, m_{H_{j}}}$ versus the maximum of the masses, $\max \left(m_{H_{i}}\right)$. The color bar indicates the strength of the phase transition for points providing an SFOEWPT. As observed in the discussion of the N2HDM T1 the minimal mass gap is already reduced through the requirement of compatibility with the EW precision data and even more by an SFOEWPT. In the N2HDM T2 an even more reduced mass gap with $\Delta_{m_{H_{i}}, m_{H_{j}}}$ below $\mathcal{O}(40 \mathrm{GeV})$ is favored by an SFOEWPT, so that at least one pair of the Higgs bosons has to be almost mass degenerate. Additionally, except for some parameter points, the mass spectrum is not too heavy with $\max \left(m_{H_{i}}\right)$ below $\mathcal{O}(750 \mathrm{GeV})$. The heavy Higgs bosons with masses above $\sim 800 \mathrm{GeV}$ are again singlet-like states. In figure $8(\mathrm{~b})$ the mass gap between $A$ and $H^{ \pm}$versus the mass gap between $H_{\downarrow}$ and $H_{\uparrow}$ is shown. Unlike the results in the N2HDM T1, we do not find the separation into the two distinct parameter regions $\mathcal{M}_{\text {deg }}$ and $\mathcal{M}_{\text {sep }}$. In the N2HDM T2, only parameter points with nearly mass degenerate $A$ and $H^{ \pm}$fulfill the experimental and theoretical constraints and simultaneously provide an SFOEWPT.

In figure 9 we show the singlet admixture $\Sigma$ of the CP-even neutral Higgs bosons $H_{\downarrow}$ (left) and $H_{\uparrow}$ (right) versus their respective mass values. The blue dashed line indicates the SM mass of $m_{h}=125.09 \mathrm{GeV}$. For both $H_{\downarrow}$ and $H_{\uparrow}$ the requirement of an SFOEWPT reduces their mass values as follows

$$
\begin{aligned}
& m_{H_{\downarrow}} \in[31,1469] \mathrm{GeV} \stackrel{\text { SFOEWPT }}{\longrightarrow}[40,493] \mathrm{GeV} \\
& m_{H_{\uparrow}} \in[387,1500] \mathrm{GeV} \stackrel{\text { SFOEWPT }}{\longrightarrow}[387,1124] \mathrm{GeV} .
\end{aligned}
$$

As observed in the N2HDM T1 masses $m_{H_{\uparrow}}$ of the order $\mathcal{O}(1 \mathrm{TeV})$ can only be realised for a singlet-like $H_{\uparrow}$ with a singlet-admixture of at least $\sim 80 \%$. The semi-inverted mass hierarchy with $m_{H_{\downarrow}} \lesssim m_{h}$ is possible in the N2HDM T2, but only for singlet-like $H_{\downarrow}$ with $\Sigma_{H_{\downarrow}}^{S} \gtrsim 92 \%$ whereas the inverted mass hierarchy is not realized. In figure $10 m_{H^{ \pm}}$is plotted 


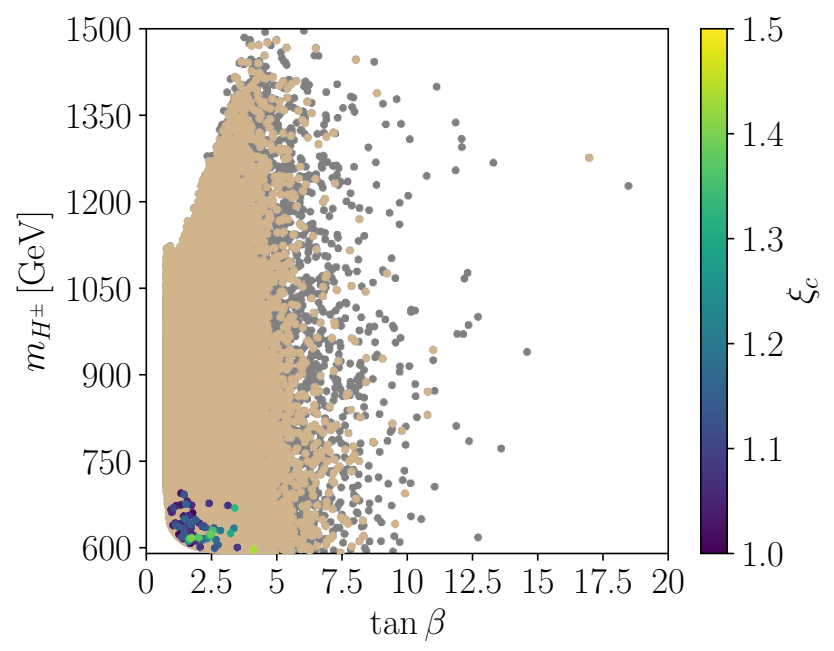

Figure 10. N2HDM T2: the charged mass $m_{H^{ \pm}}$versus $\tan \beta$. The color code is the same as in figure 3. The color bar indicates the strength of the phase transition for $\xi_{c} \geq 1$. The di-Higgs search constraints are included.

versus $\tan \beta$. As can be inferred from the figure, the requirement of an SFOEWPT reduces the upper bound of the charged mass quite significantly,

$$
m_{H^{ \pm}} \in[592,1480] \mathrm{GeV} \stackrel{\text { SFOEWPT }}{\longrightarrow}[597,694] \mathrm{GeV} .
$$

Additionally, the SFOEWPT favors small values of $\tan \beta$ in the range of

$$
\left.\tan \beta\right|_{\text {SFOEWPT }} \in[0.98,4.11] .
$$

Due to the favored intermediate mass regions for the charged mass, upcoming flavor constraints updates pushing the constraint on the charged mass higher might have an important constraining power on the N2HDM T2 with respect to an SFOEWPT as already observed in the C2HDM T2.

\subsubsection{N2HDM T2 - trilinear Higgs self-couplings}

In figure 11 the NLO trilinear coupling of the SM-like Higgs boson normalised to the NLO SM value is displayed versus the corresponding LO ratio. The requirement of an SFOEWPT reduces the range allowed by the theoretical and experimental constraints as follows,

$$
\lambda_{h h h}^{\mathrm{NLO}} / \lambda_{h h h}^{\mathrm{NLO}, \mathrm{SM}} \in[-1.92,1.99] \stackrel{\mathrm{SFOEWPT}}{\longrightarrow}[-1.92,-1.52] \cup[1.58,1.98] .
$$

As already mentioned in the discussion of the N2HDM T1, the interplay of the requirement of large quartic couplings and small to medium Higgs boson masses, pushes the trilinear couplings to enhanced values, but their upper limit remains under the allowed possibilities concerning theoretical and experimental constraints. In contrast to the N2HDM T1, in the N2HDM T2 trilinear couplings of at least 1.52 times the SM value are required for an SFOEWPT, the SM size is not sufficient. 


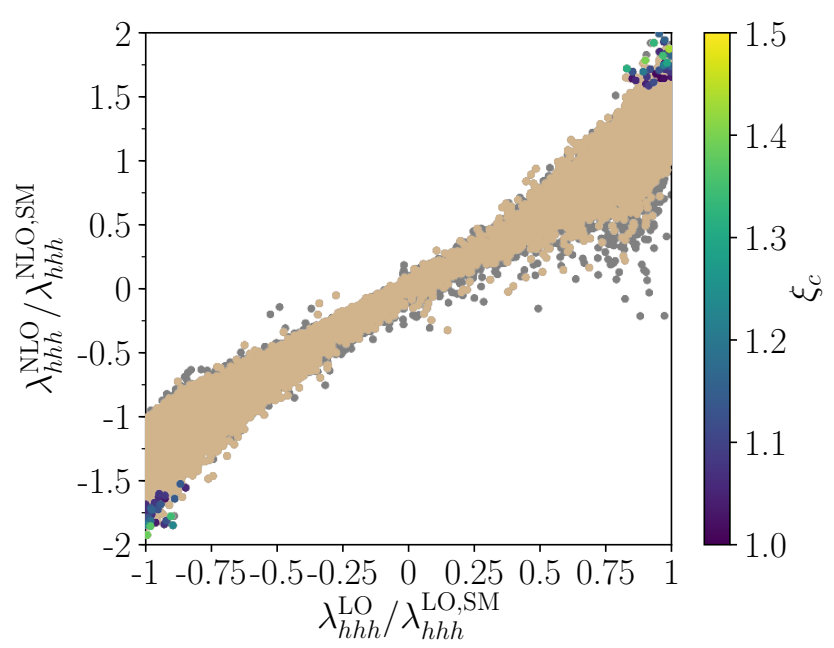

Figure 11. N2HDM T2: NLO trilinear self-coupling between three SM-like Higgs bosons normalised to the SM reference value versus the corresponding LO ratio. The color code is the same as in figure 3. The color bar indicates the strength of the phase transition for $\xi_{c} \geq 1$. The di-Higgs search constraints are included.

\subsection{Comparison of the C2HDM and the N2HDM di-Higgs rates}

So far we discussed both models independently with respect to their phenomenology of the mass spectra and the trilinear Higgs self-couplings. In the following we will compare the two models with respect to their expected signal rates in the di-Higgs final states. Both models feature three neutral Higgs bosons $\left(h, H_{\downarrow}, H_{\uparrow}\right)$ and one charged Higgs boson. Recent di-Higgs searches in the final states $4 b[129,130],(2 b)(2 \tau)[131,132]$ and $(2 b)(2 \gamma)[133]$ put strict constraints on the viable parameter space of both models so that we want to investigate to which extend future studies of this kind might help to tighten the viable parameter space of the N2HDM and C2HDM. All rates given in the following have been computed for $\sqrt{s}=13 \mathrm{TeV}$.

\subsubsection{C2HDM and N2HDM T1}

$(\overline{\boldsymbol{b}} \boldsymbol{b})(\overline{\boldsymbol{b}} \boldsymbol{b})$ final state. We start the comparison for the $(4 b)$ final state of the T1 models. In figure 12 we show the signal rates for the process $p p \rightarrow H_{\uparrow} \rightarrow\left(H_{\downarrow} \rightarrow \bar{b} b\right)\left(H_{\downarrow} \rightarrow \bar{b} b\right)$ versus the mass of the heaviest neutral Higgs boson $H_{\uparrow}$ for the C2HDM (left) and the N2HDM (right). Figure 13 displays the signal rates $p p \rightarrow H_{\uparrow} \rightarrow(h \rightarrow \bar{b} b)(h \rightarrow \bar{b} b)$ versus $m_{H_{\uparrow}}$. The rates decrease with increasing mass $m_{H_{\uparrow}}$. In figure 12 (left) a strong reduction of the C2HDM rates can be observed at the threshold for the decay into a top-quark pair, $m_{H_{\uparrow}}=350 \mathrm{GeV}$, down from $\mathcal{O}(1 \mathrm{pb})$ by more than three orders of magnitude. ${ }^{6}$ In the N2HDM, no such reduction appears as the addition of the singlet to the Higgs sector allows for singlet-like $H_{\uparrow}$ granting Higgs-to-Higgs decays being of the order $\mathcal{O}(1 \mathrm{pb})$. Reaching $m_{H_{\uparrow}} \approx 700 \mathrm{GeV}$ also $H_{\downarrow}$ can be heavier than $350 \mathrm{GeV}$ so that the decays $H_{\downarrow} \rightarrow t \bar{t}$

\footnotetext{
${ }^{6}$ Where necessary to show certain effect, we display rates down to $10^{-10}$ in the plots. Otherwise, the plots are cut at $10^{-4}$.
} 


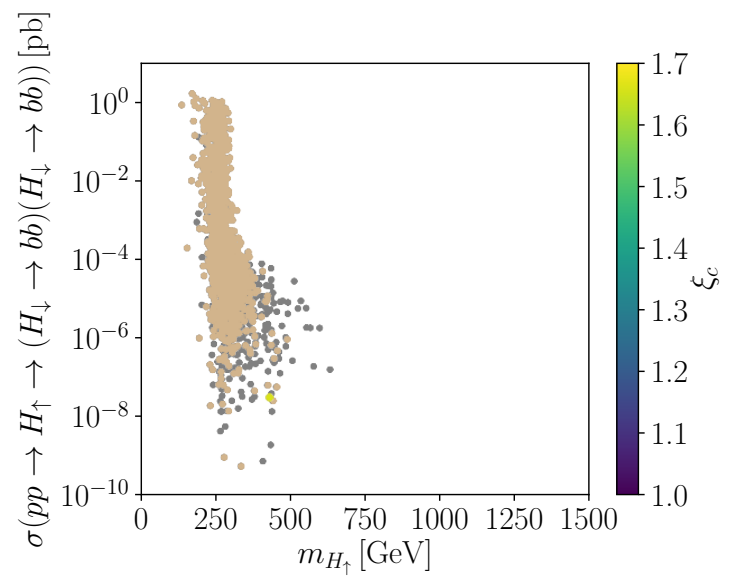

(a) C2HDM T1

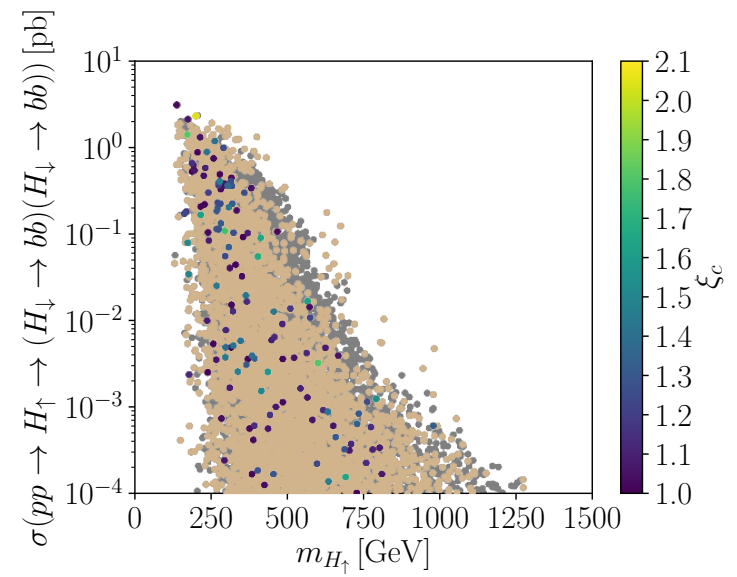

(b) N2HDM T1

Figure 12. T1: production rate of $H_{\uparrow}$ with subsequent decay into $H_{\downarrow} H_{\downarrow}$ in the $4 b$ final state versus $m_{H_{\uparrow}}$ for the C2HDM (left) and the N2HDM (right). The color code is the same as in figure 3. The color bar indicates the strength of the phase transition for $\xi_{c} \geq 1$. The di-Higgs search constraints are included.

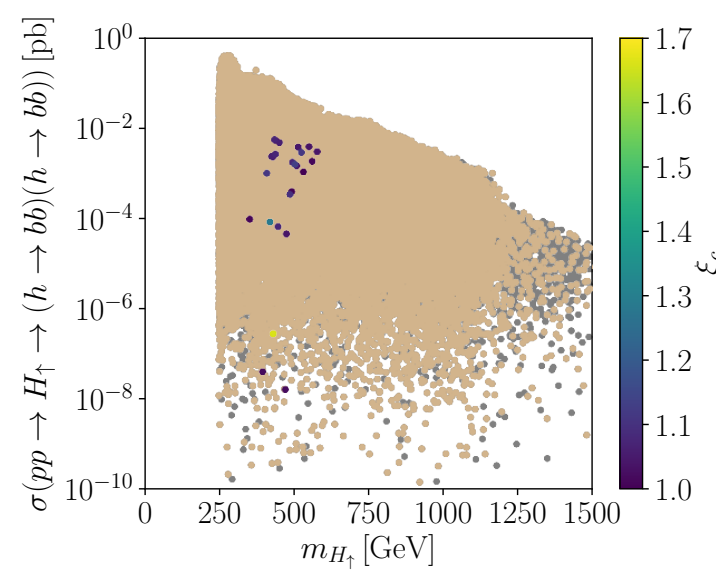

(a) C2HDM T1

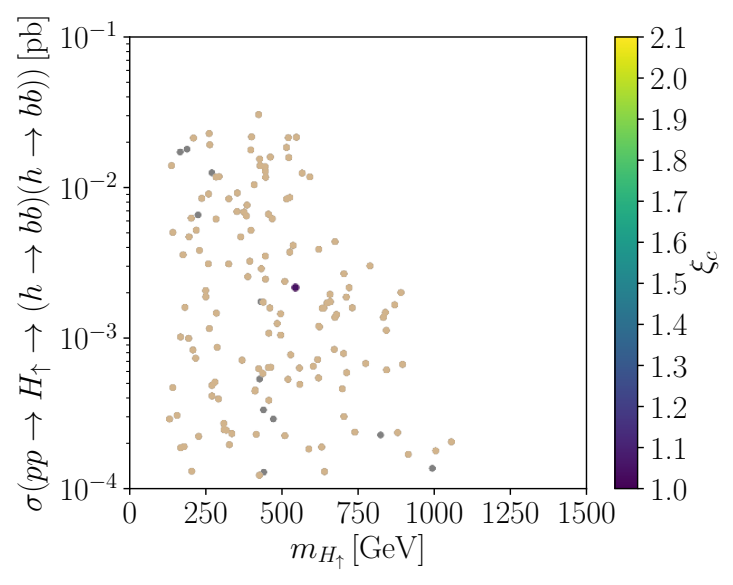

(b) N2HDM T1

Figure 13. T1: production rate of $H_{\uparrow}$ with subsequent decay into $h h$ in the $4 b$ final state versus $m_{H_{\uparrow}}$ for the C2HDM (left) and the N2HDM (right). The color code is the same as in figure 3 . The color bar indicates the strength of the phase transition for $\xi_{c} \geq 1$. The di-Higgs search constraints are included.

can become possible. In the $\mathrm{C} 2 \mathrm{HDM}$, this possibility reduces the overall cross section so effectively that no Higgs-to-Higgs decays above $\mathcal{O}\left(10^{-10} \mathrm{pb}\right)$ are realized in the C2HDM for masses above $700 \mathrm{GeV}$. With respect to a successful SFOEWPT the differences of both models are significant. In the C2HDM the requirement of an SFOEWPT reduces the maximal signal rate

$$
\max \sigma\left(p p \rightarrow H_{\uparrow} \rightarrow\left(H_{\downarrow} \rightarrow \bar{b} b\right)\left(H_{\downarrow} \rightarrow \bar{b} b\right)\right)_{\mathrm{C} 2 \mathrm{HDM}}=1611 \mathrm{fb}
$$




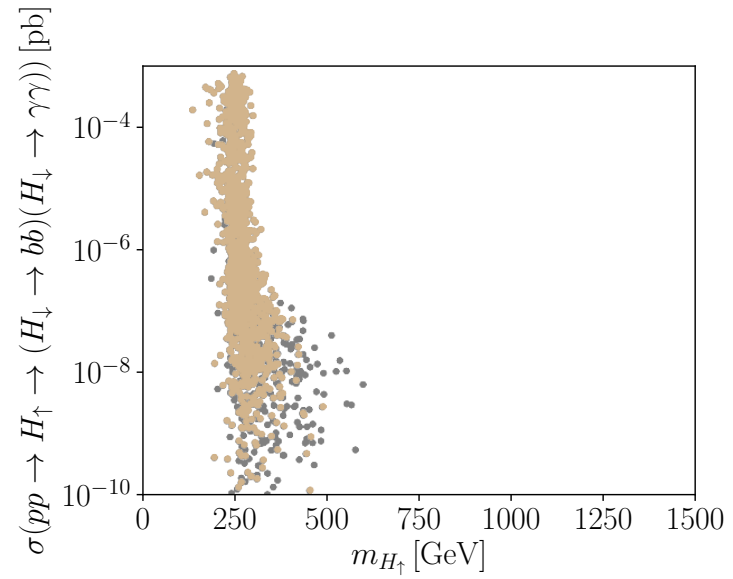

(a) C2HDM T1

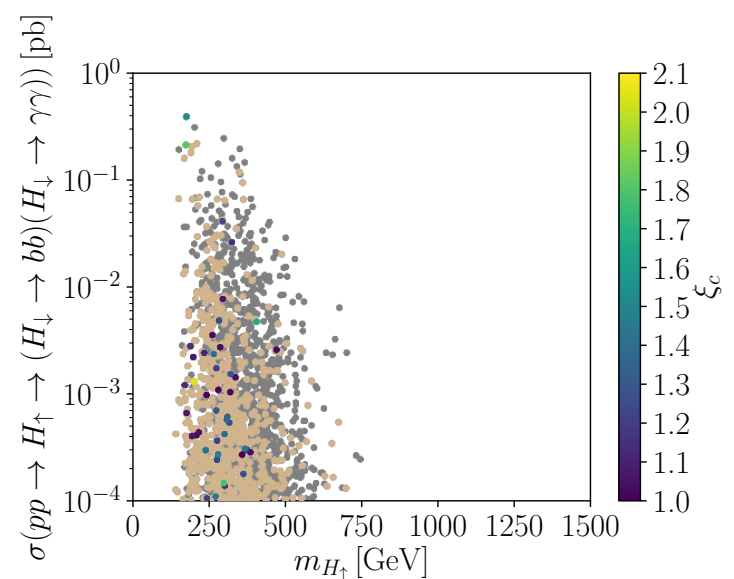

(b) N2HDM T1

Figure 14. T1: production rate of $H_{\uparrow}$ with subsequent decay into $H_{\downarrow} H_{\downarrow}$ in the $(2 b)(2 \gamma)$ final state versus $m_{H_{\uparrow}}$ for the C2HDM (left) and the N2HDM (right). The color code is the same as in figure 3. The color bar indicates the strength of the phase transition for $\xi_{c} \geq 1$. The di-Higgs search constraints are included.

to a signal rate of the oder $\mathcal{O}\left(10^{-8} \mathrm{pb}\right)$, while no such reduction is observed in the N2HDM. The SFOEWPT parameter sample covers almost the full sample and we have the following maximum rates both with and without an SFOEWPT

$$
\max \sigma\left(p p \rightarrow H_{\uparrow} \rightarrow\left(H_{\downarrow} \rightarrow \bar{b} b\right)\left(H_{\downarrow} \rightarrow \bar{b} b\right)\right)_{\mathrm{N} 2 \mathrm{HDM}}=3070 \mathrm{fb}
$$

An analogous behaviour of the maximum rates is observed in figure 13 in the decay channel

$$
\begin{aligned}
& H_{\uparrow} \rightarrow(h \rightarrow \bar{b} b)(h \rightarrow \bar{b} b) \\
& \quad \max \sigma\left(p p \rightarrow H_{\uparrow} \rightarrow(h \rightarrow \bar{b} b)(h \rightarrow \bar{b} b)\right)_{\mathrm{C} 2 \mathrm{HDM}}=385.92 \mathrm{fb} \stackrel{\text { SFOEWPT }}{\longrightarrow} 5.24 \mathrm{fb} \\
& \max \sigma\left(p p \rightarrow H_{\uparrow} \rightarrow(h \rightarrow \bar{b} b)(h \rightarrow \bar{b} b)\right)_{\mathrm{N} 2 \mathrm{HDM}}=413.20 \mathrm{fb} \stackrel{\text { SFOEWPT }}{\longrightarrow} 285.70 \mathrm{fb} .
\end{aligned}
$$

In the SM-like decay chain $H_{\uparrow} \rightarrow h h \rightarrow X X$ (and also in the decay chain $H_{\uparrow} \rightarrow H_{\downarrow} H_{\downarrow} \rightarrow$ $X X)$ increasing sensitivity in the di-Higgs searches for intermediate Higgs boson masses (up to $500 \mathrm{GeV}$ ) could allow for probing valid N2HDM candidates with a successful strong first order EWPT, in contrast to the C2HDM. In the C2HDM the cross section for the SFOEWPT parameter points is orders of magnitudes below the LHC sensitivity. Since in C2HDM and N2HDM T1 the couplings of the Higgs bosons to the down-type quarks and leptons are the same $[64,65]$ the rates in the $(b \bar{b})(\tau \tau)$ final state are simply reduced by about a factor 10 without changing the qualitative behaviour so that we do not show separate plots for this final state.

$(\overline{\boldsymbol{b}} \boldsymbol{b})(\gamma \gamma)$ final state. The rates for the $(b b)(\gamma \gamma)$ final states from $H_{\uparrow}$ production with subsequent decay into $H_{\downarrow} H_{\downarrow}$ are shown in figure 14 and those from $H_{\uparrow} \rightarrow h h$ in figure 15 . We observe the same behaviour as in the final state $(\bar{b} b)(\bar{b} b)$ with the difference that the 


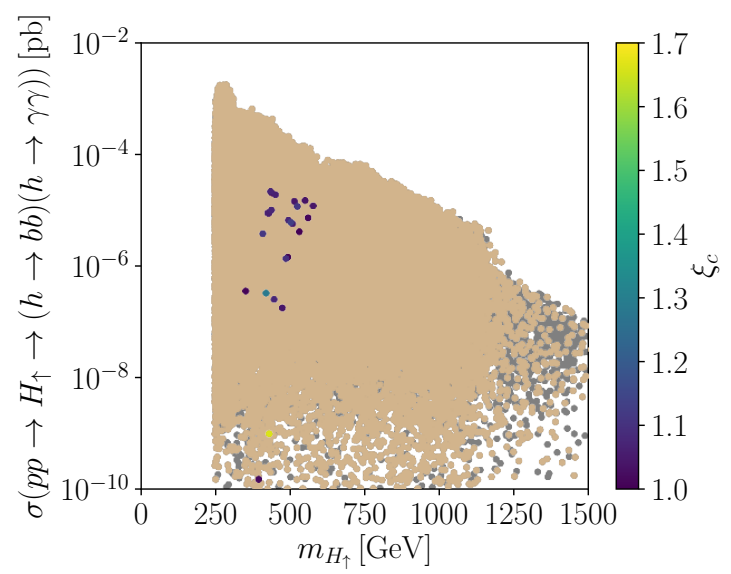

(a) C2HDM T1

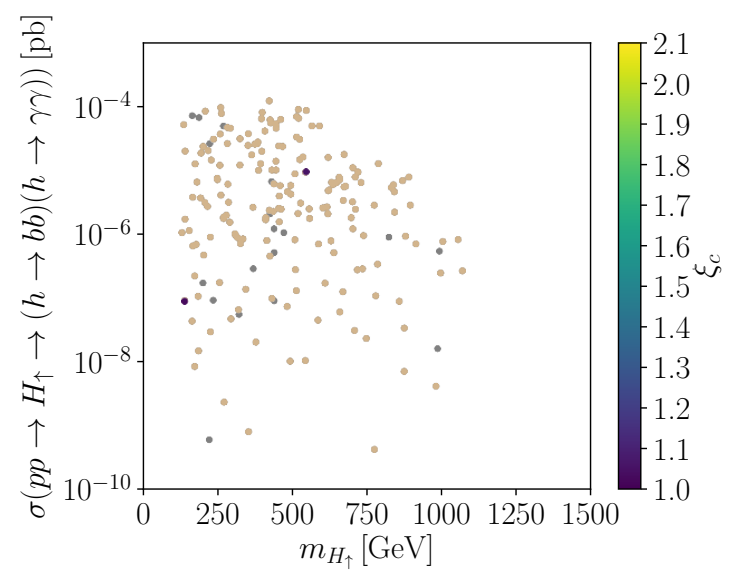

(b) N2HDM T1

Figure 15. T1: production rate of $H_{\uparrow}$ with subsequent decay into $h h$ in the $(2 b)(2 \gamma)$ final state versus $m_{H_{\uparrow}}$ for the C2HDM (left) and the N2HDM (right). The color code is the same as in figure 3. The color bar indicates the strength of the phase transition for $\xi_{c} \geq 1$. The di-Higgs search constraints are included.

overall signal strength is smaller due to the smaller branching ratio for the Higgs decays into photons. Again, in the $\mathrm{C} 2 \mathrm{HDM}$ the signal rate is significantly reduced by requiring an SFOEWPT whereas this is not the case in the N2HDM. The top threshold reduces the overall signal in the $\mathrm{C} 2 \mathrm{HDM}$ while again the $\mathrm{N} 2 \mathrm{HDM}$ is not affected due to the possibility of the singlet admixture.

\subsubsection{C2HDM and N2HDM T2}

We now turn to the comparison of the C2HDM and N2HDM T2 models. We display the signal rates $\sigma\left(p p \rightarrow H_{\uparrow} \rightarrow(h \rightarrow \bar{b} b)(h \rightarrow \bar{b} b)\right)$ versus $m_{H_{\uparrow}}$ in figure 16 . In the left plot, the results for the $\mathrm{C} 2 \mathrm{HDM}$ are shown and in the right plot those for the N2HDM. The signal rates obtained in the N2HDM and C2HDM T2 are smaller than in T1 of the models. The maximal signal rates with an SFOEWPT are

$$
\begin{aligned}
& \left.\max \sigma\left(p p \rightarrow H_{\uparrow} \rightarrow(h \rightarrow \bar{b} b)(h \rightarrow \bar{b} b)\right)\right|_{\mathrm{C} 2 \mathrm{HDM}}=0.67 \mathrm{fb} \\
& \left.\max \sigma\left(p p \rightarrow H_{\uparrow} \rightarrow(h \rightarrow \bar{b} b)(h \rightarrow \bar{b} b)\right)\right|_{\mathrm{N} 2 \mathrm{HDM}}=43.24 \mathrm{fb} .
\end{aligned}
$$

Although the N2HDM still provides larger signal rates than the C2HDM the differences are not as significant as in T1. Since we do not have parameter points with the mass relation $2 m_{H_{\downarrow}} \lesssim m_{H_{\uparrow}}$ in the C2HDM T2, we cannot discuss the comparison of the decay channel $H_{\uparrow} \rightarrow H_{\downarrow} H_{\downarrow}$ in the T2. Furthermore, the rates for the decay channel $H_{\uparrow} \rightarrow H_{\downarrow} h$ are not sufficiently enhanced to be interesting for di-Higgs searches so that we do not discuss these final states further here. The rates for the $(b \bar{b})(\gamma \gamma)$ final states are below $1 \mathrm{fb}$ in both models so that we also do not consider these channels here. 


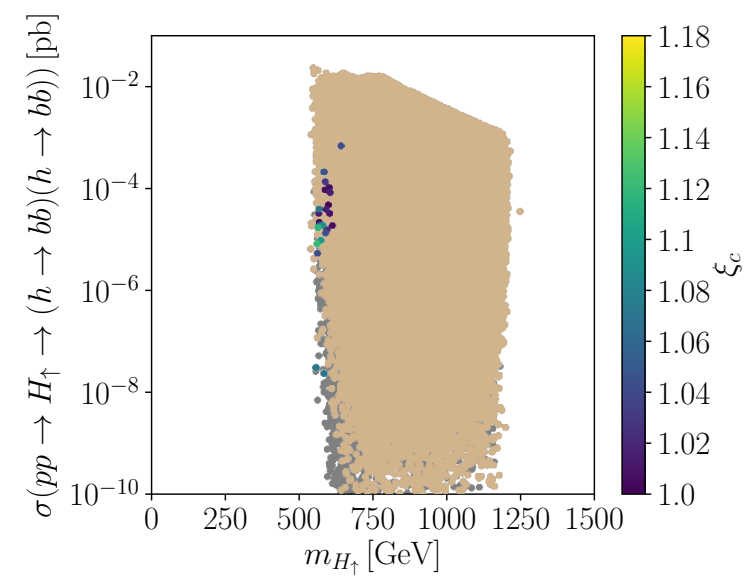

(a) C2HDM T2

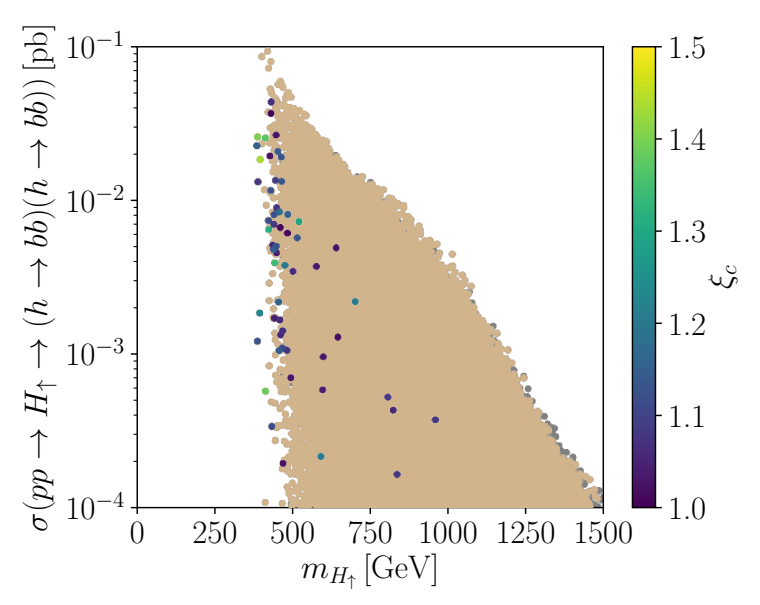

(b) N2HDM T2

Figure 16. T2: production rate of $H_{\uparrow}$ with subsequent decay into $h h$ in the $(b \bar{b})(b \bar{b})$ final state versus $m_{H_{\uparrow}}$ for the C2HDM (left) and the N2HDM (right). The color code is the same as in figure 3. The color bar indicates the strength of the phase transition for $\xi_{c} \geq 1$. The di-Higgs search constraints are included.

We conclude by remarking that the signal $\sigma\left(p p \rightarrow h \rightarrow\left(H_{\downarrow} \rightarrow \bar{b} b\right)\left(H_{\downarrow} \rightarrow \bar{b} b\right)\right)$ in the N2HDM T2 can reach

$$
\left.\max \sigma\left(p p \rightarrow h \rightarrow\left(H_{\downarrow} \rightarrow \bar{b} b\right)\left(H_{\downarrow} \rightarrow \bar{b} b\right)\right)\right|_{\mathrm{N} 2 \mathrm{HDM}}=704 \mathrm{fb} .
$$

To enable such decays a semi-inverted mass hierarchy is required where $m_{h} \gtrsim 2 m_{H_{\downarrow}}$. (The inverted mass hierarchy is not realised in the N2HDM T2.) While the signal rate is large, it has to be kept in mind, that this mass scenario is already under pressure to fulfill all required constraints.

\section{Conclusions}

In this paper, we investigated the possibility of an SFOEWPT in models with non-minimal Higgs sectors, the C2HDM and the N2HDM. For the C2HDM we updated our analysis [45] by allowing for heavier neutral scalar masses up to $1.5 \mathrm{TeV}$ and including the most recent collider constraints. Still similar mass regions compatible with theoretical and experimental constraints and the requirement of an SFOEWPT were found. The inclusion of the new constraints from the Higgs data significantly reduced, however, the scenarios compatible with an SFOEWPT. The strength of the phase transition $\xi_{c}$ in both types of the C2HDM is rather small $\left(\max \xi_{c}^{\mathrm{C} 2 \mathrm{HDM}, \mathrm{T} 1}=1.7, \max \xi_{c}^{\mathrm{C} 2 \mathrm{HDM}, \mathrm{T} 2}=1.18\right)$. In the N2HDM the inclusion of the additional real scalar singlet slightly enhances the strength of the phase transition $\left(\max \xi_{c}^{\mathrm{N} 2 \mathrm{HDM}, \mathrm{T} 1}=2.1, \max \xi_{c}^{\mathrm{N} 2 \mathrm{HDM}, \mathrm{T} 2}=1.5\right)$ compared to the C2HDM. The compatibility with the EW precision constraints requires two of the Higgs boson masses in the spectrum to be close. The additional requirement of an SFOEWPT reduces this mass gap further. For the N2HDM, we found that not all mass hierarchies were compatible both with the 
theoretical and experimental constraints and an SFOEWPT. Thus, the inverted mass hierarchy $\left(m_{H_{\downarrow}}<m_{H_{\uparrow}}<m_{h}\right)$ was not found in the N2HDM T1 and T2. In the N2HDM T2 the interplay of the requirement of small mass gaps and a heavy charged Higgs boson mass yields an overall heavier Higgs boson mass spectrum than in the N2HDM T1. We showed that the SFOEWPT favors two different mass configurations in the N2HDM T1. The first region $\mathcal{M}_{\text {deg }}$ features parameter points with $m_{A} \approx m_{H^{ \pm}}$, in the second one, $\mathcal{M}_{\text {sep }}$, the pseudoscalar Higgs boson $A$ is significantly heavier than the charged Higgs boson $H^{ \pm}$. In particular the mass region $\mathcal{M}_{\text {sep }}$ is often neglected in the context of electroweak baryogenesis. For simplicity, it is often assumed that $m_{A} \approx m_{H^{ \pm}}$. In the N2HDM T2 only points in $\mathcal{M}_{\text {deg }}$ were found to be compatible with an SFOEWPT.

We furthermore investigated in both models the trilinear Higgs self-couplings between neutral Higgs bosons. An SFOEWPT favors enhanced trilinear self-couplings. Their values remain, however, significantly below the values allowed by the experimental and theoretical constraints. For the Type I of both models they are significantly constrained by the recent updates in the di-Higgs searches. Since the SFOEWPT favors Higgs boson masses in the range $\sim 400-600 \mathrm{GeV}$, which is also the most sensitive region in the di-Higgs searches, a large part of our previous sample of parameter points providing an SFOEWPT is excluded. Future updates in the di-Higgs searches will therefore have a significant impact on the valid parameter space of the N2HDM and C2HDM with respect to an SFOEWPT. On the other hand, Type II of both models is strongly constrained due to the results for $B \rightarrow X_{s} \gamma[107-111]$. We observed that the charged Higgs mass tends to smaller mass regions if an SFOEWPT is required. This cannot be realized in Type II as the charged Higgs mass must be above $580 \mathrm{GeV}$ because of the $B \rightarrow X_{s} \gamma$ constraints. Most of the valid parameter points in the C2HDM T2 in figure 1 and in the N2HDM T2 in figure 10 are located on the left edge in the $m_{H^{ \pm}}-\tan \beta$ plane. Exactly this region is sensitive to the $B \rightarrow X_{s} \gamma$ constraints. We therefore expect the strongest constraints for the Type II to come from future updates on the charged Higgs mass constraint in the flavor sector. We finally investigated the phenomenology of both models with respect to the expected di-Higgs signals at the LHC. Requiring an SFOEWPT, the expected di-Higgs signals in the C2HDM are significantly suppressed compared to the N2HDM.

\section{Acknowledgments}

The research of MM was supported by the Deutsche Forschungsgemeinschaft (DFG, German Research Foundation) under grant 396021762 - TRR 257. P.B. acknowledges financial support by the Graduiertenkolleg GRK 1694: Elementarteilchenphysik bei höchster Energie und höchster Präzision.

Open Access. This article is distributed under the terms of the Creative Commons Attribution License (CC-BY 4.0), which permits any use, distribution and reproduction in any medium, provided the original author(s) and source are credited. 


\section{References}

[1] ATLAS collaboration, Observation of a new particle in the search for the Standard Model Higgs boson with the ATLAS detector at the LHC, Phys. Lett. B 716 (2012) 1 [arXiv:1207.7214] [INSPIRE].

[2] CMS collaboration, Observation of a New Boson at a Mass of $125 \mathrm{GeV}$ with the CMS Experiment at the LHC, Phys. Lett. B 716 (2012) 30 [arXiv:1207.7235] [InSPIRE].

[3] ATLAS collaboration, Study of the spin and parity of the Higgs boson in diboson decays with the ATLAS detector, Eur. Phys. J. C 75 (2015) 476 [Erratum ibid. C 76 (2016) 152] [arXiv: 1506.05669] [INSPIRE].

[4] CMS collaboration, Constraints on the spin-parity and anomalous $H V V$ couplings of the Higgs boson in proton collisions at 7 and 8 TeV, Phys. Rev. D 92 (2015) 012004 [arXiv: 1411.3441] [INSPIRE].

[5] ATLAS collaboration, Measurements of the Higgs boson production and decay rates and coupling strengths using pp collision data at $\sqrt{s}=7$ and 8 TeV in the ATLAS experiment, Eur. Phys. J. C 76 (2016) 6 [arXiv:1507.04548] [InSPIRE].

[6] CMS collaboration, Precise determination of the mass of the Higgs boson and tests of compatibility of its couplings with the standard model predictions using proton collisions at 7 and 8 TeV, Eur. Phys. J. C 75 (2015) 212 [arXiv:1412.8662] [InSPIRE].

[7] WMAP collaboration, Nine-Year Wilkinson Microwave Anisotropy Probe (WMAP) Observations: Final Maps and Results, Astrophys. J. Suppl. 208 (2013) 20 [arXiv: 1212.5225] [INSPIRE].

[8] V.A. Kuzmin, V.A. Rubakov and M.E. Shaposhnikov, On the Anomalous Electroweak Baryon Number Nonconservation in the Early Universe, Phys. Lett. B 155 (1985) 36 [INSPIRE].

[9] A.G. Cohen, D.B. Kaplan and A.E. Nelson, Baryogenesis at the weak phase transition, Nucl. Phys. B 349 (1991) 727 [inSPIRE].

[10] A.G. Cohen, D.B. Kaplan and A.E. Nelson, Progress in electroweak baryogenesis, Ann. Rev. Nucl. Part. Sci. 43 (1993) 27 [hep-ph/9302210] [INSPIRE].

[11] M. Quirós, Field theory at finite temperature and phase transitions, Helv. Phys. Acta 67 (1994) 451 [INSPIRE].

[12] V.A. Rubakov and M.E. Shaposhnikov, Electroweak baryon number nonconservation in the early universe and in high-energy collisions, Usp. Fiz. Nauk 166 (1996) 493 [hep-ph/9603208] [INSPIRE].

[13] K. Funakubo, CP violation and baryogenesis at the electroweak phase transition, Prog. Theor. Phys. 96 (1996) 475 [hep-ph/9608358] [InSPIRE].

[14] M. Trodden, Electroweak baryogenesis, Rev. Mod. Phys. 71 (1999) 1463 [hep-ph/9803479] [INSPIRE].

[15] W. Bernreuther, CP violation and baryogenesis, Lect. Notes Phys. 591 (2002) 237 [hep-ph/0205279] [INSPIRE].

[16] D.E. Morrissey and M.J. Ramsey-Musolf, Electroweak baryogenesis, New J. Phys. 14 (2012) 125003 [arXiv: 1206.2942] [InSPIRE].

[17] A.D. Sakharov, Violation of CP Invariance, $C$ asymmetry and baryon asymmetry of the universe, Pisma Zh. Eksp. Teor. Fiz. 5 (1967) 32 [INSPIRE]. 
[18] N.S. Manton, Topology in the Weinberg-Salam Theory, Phys. Rev. D 28 (1983) 2019 [INSPIRE].

[19] F.R. Klinkhamer and N.S. Manton, A Saddle Point Solution in the Weinberg-Salam Theory, Phys. Rev. D 30 (1984) 2212 [inSPIRE].

[20] K. Kajantie, K. Rummukainen and M.E. Shaposhnikov, A Lattice Monte Carlo study of the hot electroweak phase transition, Nucl. Phys. B 407 (1993) 356 [hep-ph/9305345] [INSPIRE].

[21] Z. Fodor, J. Hein, K. Jansen, A. Jaster and I. Montvay, Simulating the electroweak phase transition in the SU(2) Higgs model, Nucl. Phys. B 439 (1995) 147 [hep-lat/9409017] [INSPIRE].

[22] K. Kajantie, M. Laine, K. Rummukainen and M.E. Shaposhnikov, The Electroweak phase transition: A Nonperturbative analysis, Nucl. Phys. B 466 (1996) 189 [hep-lat/9510020] [INSPIRE].

[23] K. Jansen, Status of the finite temperature electroweak phase transition on the lattice, Nucl. Phys. Proc. Suppl. 47 (1996) 196 [hep-lat/9509018] [InSPIRE].

[24] K. Kajantie, M. Laine, K. Rummukainen and M.E. Shaposhnikov, Is there a hot electroweak phase transition at $m(H)$ larger or equal to $m(W)$ ?, Phys. Rev. Lett. 77 (1996) 2887 [hep-ph/9605288] [INSPIRE].

[25] F. Csikor, Z. Fodor and J. Heitger, Endpoint of the hot electroweak phase transition, Phys. Rev. Lett. 82 (1999) 21 [hep-ph/9809291] [INSPIRE].

[26] ATLAS and CMS collaboration, Combined Measurement of the Higgs Boson Mass in pp Collisions at $\sqrt{s}=7$ and $8 \mathrm{TeV}$ with the ATLAS and CMS Experiments, Phys. Rev. Lett. 114 (2015) 191803 [arXiv: 1503.07589] [INSPIRE].

[27] M.B. Gavela, P. Hernández, J. Orloff and O. Pene, Standard model CP-violation and baryon asymmetry, Mod. Phys. Lett. A 9 (1994) 795 [hep-ph/9312215] [INSPIRE].

[28] T. Konstandin, Quantum Transport and Electroweak Baryogenesis, Phys. Usp. 56 (2013) 747 [Usp. Fiz. Nauk 183 (2013) 785] [arXiv:1302.6713] [INSPIRE].

[29] J.M. Cline, Baryogenesis, in Les Houches Summer School - Session 86: Particle Physics and Cosmology: The Fabric of Spacetime, Les Houches France (2006) [hep-ph/0609145] [INSPIRE].

[30] T.D. Lee, A Theory of Spontaneous T Violation, Phys. Rev. D 8 (1973) 1226 [InSPIRE].

[31] G.C. Branco, P.M. Ferreira, L. Lavoura, M.N. Rebelo, M. Sher and J.P. Silva, Theory and phenomenology of two-Higgs-doublet models, Phys. Rept. 516 (2012) 1 [arXiv:1106.0034] [INSPIRE].

[32] A.I. Bochkarev, S.V. Kuzmin and M.E. Shaposhnikov, Electroweak baryogenesis and the Higgs boson mass problem, Phys. Lett. B 244 (1990) 275 [INSPIRE].

[33] L.D. McLerran, M.E. Shaposhnikov, N. Turok and M.B. Voloshin, Why the baryon asymmetry of the universe is approximately $10^{-10}$, Phys. Lett. B 256 (1991) 451 [INSPIRE].

[34] A.I. Bochkarev, S.V. Kuzmin and M.E. Shaposhnikov, On the Model Dependence of the Cosmological Upper Bound on the Higgs Boson and Top Quark Masses, Phys. Rev. D 43 (1991) 369 [INSPIRE].

[35] N. Turok and J. Zadrozny, Electroweak baryogenesis in the two doublet model, Nucl. Phys. B 358 (1991) 471 [INSPIRE]. 
[36] A.G. Cohen, D.B. Kaplan and A.E. Nelson, Spontaneous baryogenesis at the weak phase transition, Phys. Lett. B 263 (1991) 86 [INSPIRE].

[37] N. Turok and J. Zadrozny, Phase transitions in the two doublet model, Nucl. Phys. B 369 (1992) 729 [INSPIRE].

[38] A.E. Nelson, D.B. Kaplan and A.G. Cohen, Why there is something rather than nothing: Matter from weak interactions, Nucl. Phys. B 373 (1992) 453 [INSPIRE].

[39] K. Funakubo, A. Kakuto and K. Takenaga, The Effective potential of electroweak theory with two massless Higgs doublets at finite temperature, Prog. Theor. Phys. 91 (1994) 341 [hep-ph/9310267] [INSPIRE].

[40] A.T. Davies, C.D. froggatt, G. Jenkins and R.G. Moorhouse, Baryogenesis constraints on two Higgs doublet models, Phys. Lett. B 336 (1994) 464 [INSPIRE].

[41] J.M. Cline, K. Kainulainen and A.P. Vischer, Dynamics of two Higgs doublet CP-violation and baryogenesis at the electroweak phase transition, Phys. Rev. D 54 (1996) 2451 [hep-ph/9506284] [INSPIRE].

[42] G.C. Dorsch, S.J. Huber and J.M. No, A strong electroweak phase transition in the 2HDM after LHC8, JHEP 10 (2013) 029 [arXiv:1305.6610] [INSPIRE].

[43] G.C. Dorsch, S.J. Huber, K. Mimasu and J.M. No, Echoes of the Electroweak Phase Transition: Discovering a second Higgs doublet through $A_{0} \rightarrow Z H_{0}$, Phys. Rev. Lett. 113 (2014) 211802 [arXiv:1405.5537] [INSPIRE].

[44] G.C. Dorsch, S.J. Huber, K. Mimasu and J.M. No, The Higgs Vacuum Uplifted: Revisiting the Electroweak Phase Transition with a Second Higgs Doublet, JHEP 12 (2017) 086 [arXiv: 1705.09186] [INSPIRE].

[45] P. Basler, M. Krause, M. Muhlleitner, J. Wittbrodt and A. Wlotzka, Strong First Order Electroweak Phase Transition in the CP-Conserving 2HDM Revisited, JHEP 02 (2017) 121 [arXiv: 1612.04086] [INSPIRE].

[46] M. Laine, M. Meyer and G. Nardini, Thermal phase transition with full 2-loop effective potential, Nucl. Phys. B 920 (2017) 565 [arXiv:1702.07479] [INSPIRE].

[47] G.C. Dorsch, S.J. Huber, K. Mimasu and J.M. No, The Higgs Vacuum Uplifted: Revisiting the Electroweak Phase Transition with a Second Higgs Doublet, JHEP 12 (2017) 086 [arXiv: 1705. 09186] [INSPIRE].

[48] J.O. Andersen et al., Nonperturbative Analysis of the Electroweak Phase Transition in the Two Higgs Doublet Model, Phys. Rev. Lett. 121 (2018) 191802 [arXiv:1711.09849] [INSPIRE].

[49] J. Bernon, L. Bian and Y. Jiang, A new insight into the phase transition in the early Universe with two Higgs doublets, JHEP 05 (2018) 151 [arXiv: 1712.08430] [INSPIRE].

[50] L. Wang, J.M. Yang, M. Zhang and Y. Zhang, Revisiting lepton-specific 2HDM in light of muon g- 2 anomaly, Phys. Lett. B 788 (2019) 519 [arXiv:1809.05857] [INSPIRE].

[51] K. Kainulainen, V. Keus, L. Niemi, K. Rummukainen, T.V.I. Tenkanen and V. Vaskonen, On the validity of perturbative studies of the electroweak phase transition in the Two Higgs Doublet model, JHEP 06 (2019) 075 [arXiv: 1904.01329] [INSPIRE].

[52] G.C. Dorsch, S.J. Huber, T. Konstandin and J.M. No, A Second Higgs Doublet in the Early Universe: Baryogenesis and Gravitational Waves, JCAP 05 (2017) 052

[arXiv: 1611.05874] [INSPIRE]. 
[53] K. Funakubo, A. Kakuto, S. Otsuki, K. Takenaga and F. Toyoda, CP violating profile of the electroweak bubble wall, Prog. Theor. Phys. 94 (1995) 845 [hep-ph/9507452] [INSPIRE].

[54] J.M. Cline, K. Kainulainen and A.P. Vischer, Dynamics of two Higgs doublet CP-violation and baryogenesis at the electroweak phase transition, Phys. Rev. D 54 (1996) 2451 [hep-ph/9506284] [INSPIRE].

[55] K. Funakubo, A. Kakuto, S. Otsuki and F. Toyoda, Explicit CP breaking and electroweak baryogenesis, Prog. Theor. Phys. 96 (1996) 771 [hep-ph/9606282] [INSPIRE].

[56] J.M. Cline and P.-A. Lemieux, Electroweak phase transition in two Higgs doublet models, Phys. Rev. D 55 (1997) 3873 [hep-ph/9609240] [INSPIRE].

[57] L. Fromme, S.J. Huber and M. Seniuch, Baryogenesis in the two-Higgs doublet model, JHEP 11 (2006) 038 [hep-ph/0605242] [INSPIRE].

[58] J.M. Cline, K. Kainulainen and M. Trott, Electroweak Baryogenesis in Two Higgs Doublet Models and B meson anomalies, JHEP 11 (2011) 089 [arXiv:1107.3559] [INSPIRE].

[59] A. Haarr, A. Kvellestad and T.C. Petersen, Disfavouring Electroweak Baryogenesis and a hidden Higgs in a CP-violating Two-Higgs-Doublet Model, arXiv:1611.05757 [INSPIRE].

[60] P. Basler, M. Mühlleitner and J. Wittbrodt, The CP-Violating 2HDM in Light of a Strong First Order Electroweak Phase Transition and Implications for Higgs Pair Production, JHEP 03 (2018) 061 [arXiv: 1711. 04097] [INSPIRE].

[61] X. Wang, F.P. Huang and X. Zhang, Gravitational wave and collider signals in complex two-Higgs doublet model with dynamical CP-violation at finite temperature, Phys. Rev. D 101 (2020) 015015 [arXiv: 1909.02978] [INSPIRE].

[62] I.F. Ginzburg, M. Krawczyk and P. Osland, Two Higgs doublet models with CP-violation, in Linear colliders. Proceedings of International Workshop on physics and experiments with future electron-positron linear colliders, LCWS 2002, Seogwipo South Korea (2002), pg. 703 [hep-ph/0211371] [INSPIRE].

[63] C.-Y. Chen, M. Freid and M. Sher, Next-to-minimal two Higgs doublet model, Phys. Rev. D 89 (2014) 075009 [arXiv: 1312.3949] [InSPIRE].

[64] M. Muhlleitner, M.O.P. Sampaio, R. Santos and J. Wittbrodt, The N2HDM under Theoretical and Experimental Scrutiny, JHEP 03 (2017) 094 [arXiv:1612.01309] [INSPIRE].

[65] D. Fontes, M. Mühlleitner, J.C. Romão, R. Santos, J.P. Silva and J. Wittbrodt, The C2HDM revisited, JHEP 02 (2018) 073 [arXiv: 1711.09419] [INSPIRE].

[66] D. Fontes, J.C. Romão and J.P. Silva, $h \rightarrow Z \gamma$ in the complex two Higgs doublet model, JHEP 12 (2014) 043 [arXiv: 1408.2534] [INSPIRE].

[67] I.P. Ivanov, Minkowski space structure of the Higgs potential in 2HDM. II. Minima, symmetries and topology, Phys. Rev. D 77 (2008) 015017 [arXiv:0710.3490] [INSPIRE].

[68] A. Barroso, P.M. Ferreira and R. Santos, Neutral minima in two-Higgs doublet models, Phys. Lett. B 652 (2007) 181 [hep-ph/0702098] [INSPIRE].

[69] P.M. Ferreira, R. Santos and A. Barroso, Stability of the tree-level vacuum in two Higgs doublet models against charge or CP spontaneous violation, Phys. Lett. B 603 (2004) 219 [Erratum ibid. B 629 (2005) 114] [hep-ph/0406231] [INSPIRE].

[70] A.W. El Kaffas, P. Osland and O.M. Ogreid, CP violation, stability and unitarity of the two Higgs doublet model, Nonlin. Phenom. Complex Syst. 10 (2007) 347 [hep-ph/0702097] [INSPIRE]. 
[71] P.M. Ferreira, M. Mühlleitner, R. Santos, G. Weiglein and J. Wittbrodt, Vacuum Instabilities in the N2HDM, JHEP 09 (2019) 006 [arXiv: 1905.10234] [INSPIRE].

[72] W.G. Hollik, G. Weiglein and J. Wittbrodt, Impact of Vacuum Stability Constraints on the Phenomenology of Supersymmetric Models, JHEP 03 (2019) 109 [arXiv:1812.04644] [INSPIRE].

[73] P. Basler and M. Mühlleitner, BSMPT (Beyond the Standard Model Phase Transitions): A tool for the electroweak phase transition in extended Higgs sectors, Comput. Phys. Commun. 237 (2019) 62 [arXiv:1803.02846] [INSPIRE].

[74] S.R. Coleman and E.J. Weinberg, Radiative Corrections as the Origin of Spontaneous Symmetry Breaking, Phys. Rev. D 7 (1973) 1888 [INSPIRE].

[75] M.E. Carrington, The Effective potential at finite temperature in the Standard Model, Phys. Rev. D 45 (1992) 2933 [inSPIRE].

[76] L. Dolan and R. Jackiw, Symmetry Behavior at Finite Temperature, Phys. Rev. D 9 (1974) 3320 [INSPIRE].

[77] M. Quirós, Finite temperature field theory and phase transitions, in Proceedings of Summer School in High-energy physics and cosmology, Trieste Italy (1998), pg. 187 [hep-ph/9901312] [INSPIRE].

[78] M. Carena, G. Nardini, M. Quirós and C.E.M. Wagner, The Baryogenesis Window in the MSSM, Nucl. Phys. B 812 (2009) 243 [arXiv:0809.3760] [INSPIRE].

[79] P.B. Arnold and O. Espinosa, The Effective potential and first order phase transitions: Beyond leading-order, Phys. Rev. D 47 (1993) 3546 [Erratum ibid. D 50 (1994) 6662] [hep-ph/9212235] [INSPIRE].

[80] R.R. Parwani, Resummation in a hot scalar field theory, Phys. Rev. D 45 (1992) 4695 [Erratum ibid. D 48 (1993) 5965] [hep-ph/9204216] [INSPIRE].

[81] A.D. Linde, Infrared Problem in Thermodynamics of the Yang-Mills Gas, Phys. Lett. B 96 (1980) 289 [INSPIRE].

[82] P.H. Ginsparg, First Order and Second Order Phase Transitions in Gauge Theories at Finite Temperature, Nucl. Phys. B 170 (1980) 388 [InSPIRE].

[83] T. Appelquist and R.D. Pisarski, High-Temperature Yang-Mills Theories and Three-Dimensional Quantum Chromodynamics, Phys. Rev. D 23 (1981) 2305 [INSPIRE].

[84] K. Farakos, K. Kajantie, K. Rummukainen and M.E. Shaposhnikov, $3-D$ physics and the electroweak phase transition: Perturbation theory, Nucl. Phys. B 425 (1994) 67 [hep-ph/9404201] [INSPIRE].

[85] M. Losada, High temperature dimensional reduction of the MSSM and other multiscalar models, Phys. Rev. D 56 (1997) 2893 [hep-ph/9605266] [INSPIRE].

[86] J.M. Cline and P.-A. Lemieux, Electroweak phase transition in two Higgs doublet models, Phys. Rev. D 55 (1997) 3873 [hep-ph/9609240] [InSPIRE].

[87] M. Laine and A. Rajantie, Lattice continuum relations for $3-D \mathrm{SU}(N)+$ Higgs theories, Nucl. Phys. B 513 (1998) 471 [hep-lat/9705003] [InSPIRE].

[88] M. Laine and K. Rummukainen, The MSSM electroweak phase transition on the lattice, Nucl. Phys. B 535 (1998) 423 [hep-lat/9804019] [INSPIRE].

[89] J.O. Andersen, Dimensional reduction of the two Higgs doublet model at high temperature, Eur. Phys. J. C 11 (1999) 563 [hep-ph/9804280] [INSPIRE]. 
[90] F. Csikor, Z. Fodor and J. Heitger, Endpoint of the hot electroweak phase transition, Phys. Rev. Lett. 82 (1999) 21 [hep-ph/9809291] [INSPIRE].

[91] M. Laine, The Renormalized gauge coupling and nonperturbative tests of dimensional reduction, JHEP 06 (1999) 020 [hep-ph/9903513] [INSPIRE].

[92] M. Laine and K. Rummukainen, Two Higgs doublet dynamics at the electroweak phase transition: A Nonperturbative study, Nucl. Phys. B 597 (2001) 23 [hep-lat/0009025] [INSPIRE].

[93] J.M. Cline, K. Kainulainen and M. Trott, Electroweak Baryogenesis in Two Higgs Doublet Models and B meson anomalies, JHEP 11 (2011) 089 [arXiv:1107.3559] [INSPIRE].

[94] M. Laine, G. Nardini and K. Rummukainen, Lattice study of an electroweak phase transition at $m_{h} \sim 126 \mathrm{GeV}, J C A P 01$ (2013) 011 [arXiv:1211.7344] [INSPIRE].

[95] T. Gorda, A. Helset, L. Niemi, T.V.I. Tenkanen and D.J. Weir, Three-dimensional effective theories for the two Higgs doublet model at high temperature, JHEP 02 (2019) 081 [arXiv: 1802.05056] [INSPIRE].

[96] J.E. Camargo-Molina, A.P. Morais, R. Pasechnik, M.O.P. Sampaio and J. Wessén, All one-loop scalar vertices in the effective potential approach, JHEP 08 (2016) 073 [arXiv: 1606. 07069] [INSPIRE].

[97] W. Buchmüller, Z. Fodor and A. Hebecker, Gauge invariant treatment of the electroweak phase transition, Phys. Lett. B 331 (1994) 131 [hep-ph/9403391] [INSPIRE].

[98] M. Laine, Gauge dependence of the high temperature two loop effective potential for the Higgs field, Phys. Rev. D 51 (1995) 4525 [hep-ph/9411252] [INSPIRE].

[99] H.H. Patel and M.J. Ramsey-Musolf, Baryon Washout, Electroweak Phase Transition and Perturbation Theory, JHEP 07 (2011) 029 [arXiv: 1101.4665] [INSPIRE].

[100] C. Wainwright, S. Profumo and M.J. Ramsey-Musolf, Gravity Waves from a Cosmological Phase Transition: Gauge Artifacts and Daisy Resummations, Phys. Rev. D 84 (2011) 023521 [arXiv: 1104.5487] [INSPIRE].

[101] C.L. Wainwright, S. Profumo and M.J. Ramsey-Musolf, Phase Transitions and Gauge Artifacts in an Abelian Higgs Plus Singlet Model, Phys. Rev. D 86 (2012) 083537 [arXiv: 1204.5464] [INSPIRE].

[102] G.D. Moore, Measuring the broken phase sphaleron rate nonperturbatively, Phys. Rev. D 59 (1999) 014503 [hep-ph/9805264] [INSPIRE].

[103] Particle Data Group collaboration, Review of Particle Physics, Chin. Phys. C 38 (2014) 090001 [INSPIRE].

[104] A. Denner at al., Standard Model input parameters for Higgs physics, LHCHXSWG-INT-2015-006 (2015).

[105] LHC Higgs Cross Section Working Group collaboration, Handbook of LHC Higgs Cross Sections: 1. Inclusive Observables, arXiv:1101.0593 [INSPIRE].

[106] H.E. Haber and H.E. Logan, Radiative corrections to the Z $b \bar{b}$ vertex and constraints on extended Higgs sectors, Phys. Rev. D 62 (2000) 015011 [hep-ph/9909335] [INSPIRE].

[107] O. Deschamps, S. Descotes-Genon, S. Monteil, V. Niess, S. T'Jampens and V. Tisserand, The Two Higgs Doublet of Type II facing flavour physics data, Phys. Rev. D 82 (2010) 073012 [arXiv: 0907.5135] [INSPIRE]. 
[108] F. Mahmoudi and O. Stal, Flavor constraints on the two-Higgs-doublet model with general Yukawa couplings, Phys. Rev. D 81 (2010) 035016 [arXiv:0907.1791] [INSPIRE].

[109] T. Hermann, M. Misiak and M. Steinhauser, $\bar{B} \rightarrow X_{s} \gamma$ in the Two Higgs Doublet Model up to Next-to-Next-to-Leading Order in QCD, JHEP 11 (2012) 036 [arXiv:1208.2788] [INSPIRE].

[110] M. Misiak et al., Updated NNLO QCD predictions for the weak radiative B-meson decays, Phys. Rev. Lett. 114 (2015) 221801 [arXiv:1503.01789] [INSPIRE].

[111] M. Misiak and M. Steinhauser, Weak radiative decays of the $B$ meson and bounds on $M_{H^{ \pm}}$ in the Two-Higgs-Doublet Model, Eur. Phys. J. C 77 (2017) 201 [arXiv:1702.04571] [INSPIRE].

[112] M.E. Peskin and T. Takeuchi, Estimation of oblique electroweak corrections, Phys. Rev. D 46 (1992) 381 [INSPIRE].

[113] W. Grimus, L. Lavoura, O.M. Ogreid and P. Osland, A Precision constraint on multi-Higgs-doublet models, J. Phys. G 35 (2008) 075001 [arXiv:0711.4022] [INSPIRE].

[114] W. Grimus, L. Lavoura, O.M. Ogreid and P. Osland, The Oblique parameters in multi-Higgs-doublet models, Nucl. Phys. B 801 (2008) 81 [arXiv:0802.4353] [INSPIRE].

[115] GFitter Group collaboration, The global electroweak fit at NNLO and prospects for the LHC and ILC, Eur. Phys. J. C 74 (2014) 3046 [arXiv: 1407.3792] [InSPIRE].

[116] R. Coimbra, M.O.P. Sampaio and R. Santos, ScannerS: Constraining the phase diagram of a complex scalar singlet at the LHC, Eur. Phys. J. C 73 (2013) 2428 [arXiv:1301.2599] [INSPIRE].

[117] P.M. Ferreira, R. Guedes, M.O.P. Sampaio and R. Santos, Wrong sign and symmetric limits and non-decoupling in 2HDMs, JHEP 12 (2014) 067 [arXiv:1409.6723] [INSPIRE].

[118] R. Costa, M. Mühlleitner, M.O.P. Sampaio and R. Santos, Singlet Extensions of the Standard Model at LHC Run 2: Benchmarks and Comparison with the NMSSM, JHEP 06 (2016) 034 [arXiv: 1512.05355] [INSPIRE].

[119] I.P. Ivanov and J.P. Silva, Tree-level metastability bounds for the most general two Higgs doublet model, Phys. Rev. D 92 (2015) 055017 [arXiv:1507.05100] [INSPIRE].

[120] P. Bechtle, O. Brein, S. Heinemeyer, G. Weiglein and K.E. Williams, HiggsBounds: Confronting Arbitrary Higgs Sectors with Exclusion Bounds from LEP and the Tevatron, Comput. Phys. Commun. 181 (2010) 138 [arXiv:0811.4169] [INSPIRE].

[121] P. Bechtle, O. Brein, S. Heinemeyer, G. Weiglein and K.E. Williams, HiggsBounds 2.0.0: Confronting Neutral and Charged Higgs Sector Predictions with Exclusion Bounds from LEP and the Tevatron, Comput. Phys. Commun. 182 (2011) 2605 [arXiv:1102.1898] [InSPIRE].

[122] P. Bechtle et al., HiggsBounds - 4: Improved Tests of Extended Higgs Sectors against Exclusion Bounds from LEP, the Tevatron and the LHC, Eur. Phys. J. C 74 (2014) 2693 [arXiv:1311.0055] [INSPIRE].

[123] P. Bechtle, S. Heinemeyer, O. Stål, T. Stefaniak and G. Weiglein, HiggsSignals: Confronting arbitrary Higgs sectors with measurements at the Tevatron and the LHC, Eur. Phys. J. C 74 (2014) 2711 [arXiv:1305.1933] [InSPIRE].

[124] I. Engeln, M. Mühlleitner and J. Wittbrodt, N2HDECAY: Higgs Boson Decays in the Different Phases of the N2HDM, Comput. Phys. Commun. 234 (2019) 256 [arXiv: 1805. 00966] [INSPIRE]. 
[125] A. Djouadi, J. Kalinowski and M. Spira, HDECAY: A Program for Higgs boson decays in the standard model and its supersymmetric extension, Comput. Phys. Commun. 108 (1998) 56 [hep-ph/9704448] [INSPIRE].

[126] A. Djouadi, J. Kalinowski, M. Muehlleitner and M. Spira, HDECAY: Twenty++ years after, Comput. Phys. Commun. 238 (2019) 214 [arXiv:1801.09506] [InSPIRE].

[127] S. Inoue, M.J. Ramsey-Musolf and Y. Zhang, CP-violating phenomenology of flavor conserving two Higgs doublet models, Phys. Rev. D 89 (2014) 115023 [arXiv:1403.4257] [INSPIRE].

[128] ACME collaboration, Improved limit on the electric dipole moment of the electron, Nature 562 (2018) 355 [INSPIRE].

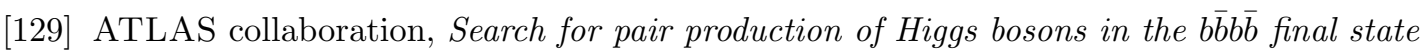
using proton-proton collisions at $\sqrt{s}=13 \mathrm{TeV}$ with the ATLAS detector, JHEP 01 (2019) 030 [arXiv: 1804.06174] [INSPIRE].

[130] CMS collaboration, Search for a massive resonance decaying to a pair of Higgs bosons in the four $b$ quark final state in proton-proton collisions at $\sqrt{s}=13$ TeV, Phys. Lett. B 781 (2018) 244 [arXiv: 1710.04960] [INSPIRE].

[131] CMS collaboration, Search for Higgs boson pair production in events with two bottom quarks and two tau leptons in proton-proton collisions at $\sqrt{s}=13$ TeV, Phys. Lett. B 778 (2018) 101 [arXiv: 1707.02909] [INSPIRE].

[132] ATLAS collaboration, Search for resonant and non-resonant Higgs boson pair production in the $b \bar{b} \tau^{+} \tau^{-}$decay channel in pp collisions at $\sqrt{s}=13 \mathrm{TeV}$ with the ATLAS detector, Phys. Rev. Lett. 121 (2018) 191801 [Erratum ibid. 122 (2019) 089901] [arXiv:1808. 00336] [INSPIRE].

[133] CMS collaboration, Search for Higgs boson pair production in the final state containing two photons and two bottom quarks in proton-proton collisions at $\sqrt{s}=13 \mathrm{TeV}$, CMS-PAS-HIG-17-008 (2017).

[134] P. Athron, C. Balázs, A. Fowlie, G. Pozzo, G. White and Y. Zhang, Strong first-order phase transitions in the NMSSM - a comprehensive survey, JHEP 11 (2019) 151 [arXiv: 1908.11847] [INSPIRE].

[135] S. Kanemura, S. Kiyoura, Y. Okada, E. Senaha and C.P. Yuan, New physics effect on the Higgs selfcoupling, Phys. Lett. B 558 (2003) 157 [hep-ph/0211308] [INSPIRE].

[136] S. Borowka et al., Higgs Boson Pair Production in Gluon Fusion at Next-to-Leading Order with Full Top-Quark Mass Dependence, Phys. Rev. Lett. 117 (2016) 012001 [Erratum ibid. 117 (2016) 079901] [arXiv: 1604.06447] [INSPIRE].

[137] S. Borowka et al., Full top quark mass dependence in Higgs boson pair production at NLO, JHEP 10 (2016) 107 [arXiv:1608.04798] [INSPIRE].

[138] J. Baglio, F. Campanario, S. Glaus, M. Mühlleitner, M. Spira and J. Streicher, Gluon fusion into Higgs pairs at NLO QCD and the top mass scheme, Eur. Phys. J. C 79 (2019) 459 [arXiv: 1811.05692] [INSPIRE]. 